\title{
Low-frequency selectivity in flat-plate boundary layer with elliptic leading edge
}

\author{
Bofu Wang ${ }^{1}$, Xuerui Mao ${ }^{1} \dagger$ and Tamer A. Zaki ${ }^{2}$ \\ ${ }^{1}$ Department of Mechanical Engineering, University of Nottingham, Nottingham NG7 2RD, UK \\ ${ }^{2}$ Department of Mechanical Engineering, Johns Hopkins University, Baltimore, MD 21218, USA
}

(Received 24 January 2018; revised 30 November 2018; accepted 27 January 2019; first published online 6 March 2019)

Linear perturbation analyses of zero-pressure-gradient boundary layers at subcritical Reynolds numbers predict that transient disturbance amplification can take place due to the lift-up mechanism. Upstream, streamwise-elongated vortices yield the largest response per unit of inflow disturbance energy, which takes the form of streamwise-elongated streaks. In this work, we compute the linear and also nonlinear inflow disturbances that generate the largest response inside the boundary layer, for flow over a thin flat plate with a slender leading edge. In order to compare our results with earlier linear analyses, we constrain the inlet disturbance to be monochromatic in time, or a single frequency. The boundary layer effectively filters high frequencies, and only low-frequency perturbations induce a strong response downstream. The low-frequency optimal inflow disturbance has a spanwise wavenumber that scales with $\sqrt{R e}$, and it consists of streamwise and normal vorticity components: the latter is tilted around the leading edge into the streamwise direction and, further downstream, generates streaks. While none of the computed monochromatic disturbances alone can lead to breakdown to turbulence, secondary instability analyses demonstrate that the streaky base state is unstable. Nonlinear simulations where the inflow disturbance is supplemented with additional white noise undergo secondary instability and breakdown to turbulence.

Key words: boundary layer receptivity, boundary layer stability, nonlinear instability

\section{Introduction}

Bypass transition refers to the evolution of a boundary-layer flow from laminar to turbulent in response to external forcing with perturbation intensities larger than $0.5 \%$ of the free-stream speed. The early stages involve the interaction of the external perturbations with the laminar flow profile - an interaction that gives rise to amplifying velocity streaks. These structures are elongated in the downstream direction, and are dominated by the streamwise velocity perturbation. They reach much larger amplitudes than the free-stream forcing that led to their genesis and, ultimately, undergo secondary instability and become hosts to localized turbulence

$\dagger$ Email address for correspondence: maoxuerui@sina.com 
spots (e.g. see review by Zaki (2013)). Owing to their role in bypass transition, streaks have been studied extensively using experiments, simulations and linear theory. The theory often adopts a Blasius profile for the base state, and thus ignores the leading-edge region of the flat plate over which the boundary layer develops. The linearity assumption also implies that disturbances remain monochromatic in homogeneous dimensions, which are time and the span for two-dimensional boundary layers. In the present study, we consider the effect of the leading edge on the linear amplification of disturbances in zero-pressure-gradient boundary layers. We also consider nonlinear disturbances, but constrain the inlet spectra to be monochromatic in time in order to distinguish the roles of different frequencies within a general broadband free-stream perturbation.

\subsection{Streaks in transitional boundary layers}

Streaks in the pre-transitional boundary layer were noted by Klebanoff (1971), and at the time he referred to them as breathing modes because they were accompanied by thinning and thickening of the boundary layer. They were later termed Klebanoff streaks by Kendall (1991). They are very-low-frequency, elongated structures and are dominated by the streamwise velocity perturbation. A visualization of the perturbation field alone, after the mean has been removed, shows forward and backward streaky patterns. In the full velocity field, they are regions of higher and lower momentum than the mean flow.

The streak perturbation is a result of lift-up of mean momentum (Taylor 1939; Landahl 1975). A small positive wall-normal velocity disturbance displaces lowmomentum fluid upwards, thus creating a negative streamwise perturbation with much higher amplitude. Conversely, a negative wall-normal velocity disturbance sweeps high-momentum fluid towards the wall, and creates a positive streamwise velocity perturbation. This mechanism has been reported to be robust with respect to modifications of the base state (Brandt et al. 2011). Monokrousos et al. (2010) found that the linear lift-up mechanism is most efficient for steady disturbances and degrades slowly for increasing frequencies, in the calculation of three-dimensional optimal perturbations in a Blasius boundary layer without a leading edge. The low-frequency appearance of streaks has also been explained in terms of a low-frequency filtering effect by the mean shear (Hunt \& Durbin 1999; Zaki \& Saha 2009), and the amplification of streaks without the action of a restoring pressure (Phillips 1969). Numerically, Schrader et al. (2010) demonstrated this sheltering in the linear regime, by computing the boundary-layer response to small-amplitude inlet perturbations each with a particular wavenumber-vector orientation.

Alternative explanations have been proposed based on the linear perturbation equations for the wall-normal velocity and vorticity, or the Orr-Sommerfeld and Squire equations. The eigenspectrum of the former is stable and each mode alone is decaying. However, the vertical velocity perturbation appears as a forcing term in the evolution equation for normal vorticity. The precise form is of streamwise vorticity perturbation acting on the mean spanwise component, thus tilting it to generate wall-normal vorticity. The response can be viewed as a superposition of Squire modes, all of which are decaying, but since they are non-orthogonal their incomplete cancellation gives rise to the amplifying streaks (Hultgren \& Gustavsson 1981; Zaki \& Durbin 2005, 2006).

Linear optimal disturbance analyses have sought the most dangerous inflow condition that leads to the maximum downstream transient energy amplification 
in a boundary layer (Butler \& Farrell 1992; Andersson, Berggren \& Henningson 1999; Schmid \& Henningson 2000). The optimal inflow condition is a steady streamwise-aligned vortex, and the response is the amplifying streak, which was also observed in nonlinear calculations of optimal inlet conditions of a Blasius flow using steady boundary-layer equations (Zuccher, Bottaro \& Luchini 2006). The theory also accurately predicts the spanwise size of the perturbations to be of the order of the local boundary-layer thickness, which is consistent with experimental observations (e.g. Matsubara \& Alfredsson 2001; Nolan \& Walsh 2012), and has also been verified by results from direct numerical simulations (DNS) (e.g. Jacobs \& Durbin 2001; Nagarajan, Lele \& Ferziger 2007). The experiments by Westin et al. (1994) showed that the root mean square of the streamwise velocity perturbation, associated with the streaks, amplifies with square root of the distance from the leading edge. This scaling agrees with the theoretical prediction by Luchini (2000). A similar scaling was also reported by Matsubara \& Alfredsson (2001), and the profiles were self-similar when normalized by their maxima.

In the absence of a seed for secondary instability, the streaks would first amplify and ultimately decay due to viscosity - hence the terminology 'transient growth'. On the other hand, if the streaks are perturbed, for example by high-frequency free-stream vortical forcing, they become unstable. Their instability is followed by the formation of turbulent spots, which are localized in space but spread as they advect downstream. As spots grow in volume, they ultimately merge and form a fully turbulent boundary layer.

\subsection{Secondary instability of streaks}

An empirical view of the role of streaks in bypass transition was provided from experiments and DNS of zero-pressure-gradient boundary layers. Their secondary instability is manifest as a streamwise undulation (e.g. Matsubara \& Alfredsson 2001; Asai, Minagawa \& Nishioka 2002; Mandal, Venkatakrishnan \& Dey 2010), which can be described as sinuous or varicose depending on the spanwise symmetry. Matsubara \& Alfredsson (2001) captured the progression of streaks from amplification through instability and breakdown to turbulence in their experiments, and Mans, de Lange \& van Steenhoven (2007) provided detailed measurements of the streak sinuous instability. Very recently, Balamurugan \& Mandal (2017) studied the localized secondary instability by simultaneous orthogonal dual-plane particle image velocimetry (PIV) measurements. They found an oscillating shear layer in the wall-normal plane is associated with the sinuous or varicose streak oscillation in the spanwise plane, and they also identified a localized secondary instability wavepacket which can originate near the boundary-layer edge.

In numerical simulations, Jacobs \& Durbin (2001) noted that the low-speed streaks lift up towards the edge of the boundary layer, where they are exposed to high-frequency free-stream forcing. They subsequently develop a wavy motion, and break down to turbulence. Brandt, Schlatter \& Henningson (2004) made similar observations, and characterized the streak instability to be primarily sinuous. These observations motivated Zaki \& Durbin $(2005,2006)$ to simulate the interaction of two perturbations: a low-frequency disturbance that generates streaks, and a high-frequency one that initiates the secondary instability of the lifted low-speed streaks near the edge of the boundary layer. The simulations by Nagarajan et al. (2007) demonstrated that the streak instability can also take place near the wall, in a fashion similar to what Brandt \& de Lange (2008) later described as a streak collision. 
Whether Tollmien-Schlichting (TS) waves play a role in streak instability was also a matter of interest in a number of studies. Boiko et al. (1994) and later Westin et al. (1998) showed that the presence of TS waves can promote transition to turbulence in streaky boundary layers. This outcome is, however, far from inevitable. For example, the spanwise shear associated with low-amplitude streaks can stabilize the TS waves (Cossu \& Brandt 2002; Fransson et al. 2005); while higher-amplitude streaks can promote the secondary instability of the TS waves themselves, but at spanwise wavenumbers that match the streak spacing (Liu, Zaki \& Durbin 2008a,b). Recently, Bose \& Durbin (2016) demonstrated the presence of a new helical streak instability and drew analogy to the instabilities of jets.

Linear stability analyses were performed in order to provide a theoretical interpretation of the above observations. Andersson et al. (2001) examined the inviscid instability of nonlinear streaks, obtained from the nonlinear flow response to forcing by the linearly optimal disturbance. They predicted that the sinuous streak instability is the fastest growing. The viscous analysis by Vaughan \& Zaki (2011) demonstrated that streaks have two types of instabilities, namely outer and inner modes, which are differentiated by the height of their respective critical layers from the wall. The former class encompasses the secondary instability of lifted low-speed streaks which are destabilized when exposed to high-frequency vortical forcing from the free stream. The inner modes, on the other hand, reside nearer to the wall and induce transition in a manner that resembles the wavepackets reported by Nagarajan et al. (2007). The base streaks in the above analyses were idealized, because they are the nonlinear flow response to a monochromatic inflow disturbance. They were repeated in the span, and the resulting instability was thus a Floquet, or collective, oscillation of the entire row of streaks.

Hack \& Zaki (2014) examined the secondary instability of streaks obtained from realistic simulations of bypass transition beneath free-stream turbulence. Their base states therefore comprised a wide variety of streak shapes, orientation and amplitudes. They demonstrated that linear theory can accurately predict the localized secondary instabilities of streaks, which precede breakdown to turbulence. They also demonstrated that outer streak instabilities are most prevalent in zero-pressure-gradient boundary layers, while the inner modes are more commonly observed in an adverse pressure gradient. Their results are consistent with the work by Nolan \& Zaki (2013), who employed structure identification and tracking techniques to isolate the particular streaks that initiate transition to turbulence, and demonstrated that transition onset shifts from the edge of the boundary layer towards the wall with progressively adverse pressure gradient. Most recently, Hack \& Zaki (2016) trained a neural network to identify the unstable streaks with the same level of accuracy as linear theory, but at a fraction of the computational cost.

\subsection{Motivation}

Linear studies of streak amplification in canonical boundary layers adopted the Blasius profile as the base state, and therefore the influence of the leading edge was not considered. In addition, the linear assumption precludes the distortion of the mean profile by Reynolds stresses, and the generation of harmonics of the perturbation and saturation. It is therefore important to assess these effects on the most effective inflow disturbance, which causes the maximum response in a spatially developing boundary layer downstream of a leading edge.

The present work assesses how the leading-edge and nonlinear effects alter the selectivity process whereby low-frequency free-stream perturbations are most likely 
to penetrate the boundary layer and amplify downstream. First the influence of the leading edge will be examined when the perturbation amplitude is sufficiently small and, as a result, nonlinearity is unimportant. The inflow disturbances will be monochromatic in time and in the homogeneous cross-flow directions upstream of the leading edge, which facilitates comparison to earlier linear theory for the Blasius profile. Nonlinearity will then be introduced in the problem, although the inflow disturbance will still be restricted to be monochromatic in time (but not in the cross-flow wavenumbers). The secondary instability of the downstream, streaky boundary layer will be examined using linear theory. The results will be discussed in the context of the literature on secondary instabilities of streaks, and observations from previous simulations and experiments.

The theoretical formulation is summarized in $\S 2$, followed by the set-up of the computational domain and flow parameters in $\S 3$. The results from the linear and nonlinear analyses are detailed in $\S \S 4$ and 5, respectively. Secondary instability analyses of the distorted boundary layer and simulations of breakdown to turbulence are reported in $\S 6$. Concluding remarks are provided in $\S 7$.

\section{Governing equations and algorithms}

The flow of an incompressible fluid over a flat plate is governed by the NavierStokes (NS) equations,

$$
\partial_{t} \boldsymbol{u}+\boldsymbol{u} \cdot \nabla \boldsymbol{u}+\nabla p-R^{-1} \nabla^{2} \boldsymbol{u}=0, \quad \nabla \cdot \boldsymbol{u}=0,
$$

where $\boldsymbol{u}=(u, v, w)^{\mathrm{T}}$ and $p$ are the non-dimensional velocity vector and pressure, respectively. Here $u, v$ and $w$ are the streamwise, normal and spanwise velocity components, respectively. The Reynolds number $R e \equiv U_{\infty} R / v$ is based on the free-stream speed $\equiv U_{\infty}$, a reference length $R$ and the fluid viscosity $v$. Here $R$ is the half-thickness of the flat plate over which the boundary layer develops. The left-hand side of the momentum equations will be denoted NS. The flow variables in (2.1) can be decomposed as the sum of a base state, which in the present case is a two-dimensional and steady solution of (2.1), and a perturbation field, $(\boldsymbol{u}, p)=(\boldsymbol{U}, P)+\left(\boldsymbol{u}^{\prime}, p^{\prime}\right)$.

The inflow perturbation is dependent on both space and time,

$$
\boldsymbol{u}^{\prime}\left(\boldsymbol{x}=\boldsymbol{x}_{B}, t\right)=G(t) \boldsymbol{u}_{B}^{\prime}\left(\boldsymbol{x}_{B}\right),
$$

where $\boldsymbol{B}$ denotes the inflow boundary (see figure 1) and $\boldsymbol{u}_{B}^{\prime}\left(\boldsymbol{x}_{B}\right)$ is the spatial dependence of the inflow perturbation. The temporal dependence is given by

$$
G(t)=\left(1-\mathrm{e}^{-\sigma t^{2}}\right)\left(1-\mathrm{e}^{-\sigma(T-t)^{2}}\right) \mathrm{e}^{\mathrm{i} \omega t},
$$

where $\sigma$ is a relaxation factor set to $\sigma=100$ throughout this work and, unless otherwise stated, the final time is set to $T=180$. It was verified that further increase of $\sigma$ does not change the results presented herein. The first two terms on the right of (2.3) ensure that $\boldsymbol{u}^{\prime}\left(\boldsymbol{x}_{B}, 0\right)=0$ and $\boldsymbol{u}^{\prime}\left(\boldsymbol{x}_{B}, T\right)=0$. The last term specifies the frequency $\omega$ of the inflow perturbation. The energy of the inflow perturbation is given by the following integral, which will be referred to as the $b$-norm,

$$
\left\|\boldsymbol{u}_{\boldsymbol{B}}^{\prime}\right\|_{b}=\left(\int_{\boldsymbol{B}} \boldsymbol{u}_{\boldsymbol{B}}^{\prime} \cdot \boldsymbol{u}_{\boldsymbol{B}}^{\prime} \mathrm{d} \boldsymbol{B}\right)^{1 / 2} .
$$

Three algorithms are adopted in this work to evaluate the linear and nonlinear optimal inflow perturbations and the secondary instability of the downstream flow. They are presented in $\S \S 2.1$ to 2.3 . 

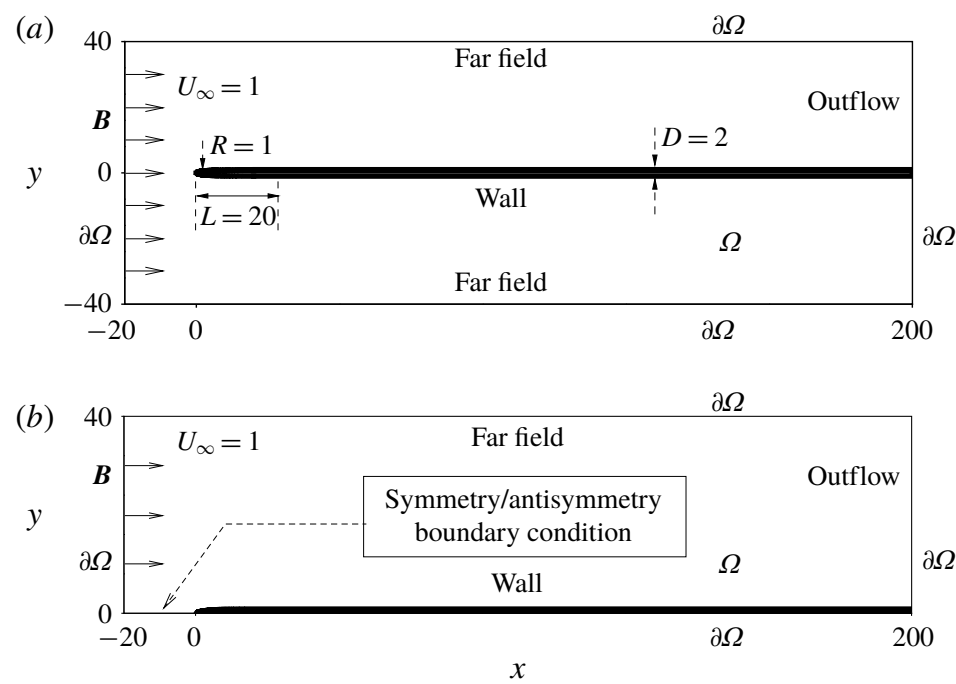

FIgURE 1. Computational domain and boundary conditions. (a) Full domain for nonlinear analysis, and $(b)$ half-domain for linear analysis.

\subsection{Nonlinear optimal inflow perturbation}

At every frequency, the objective of the analysis is to determine the inflow perturbation with a prescribed amount of energy, or $b$-norm, that is most effective at perturbing the boundary layer. The boundary-layer response is measured in terms of the perturbation energy within the flow domain at the final time $T$,

$$
E(T)=\int_{\Omega} W \boldsymbol{u}_{T}^{\prime} \cdot \boldsymbol{u}_{T}^{\prime} \mathrm{d} \Omega,
$$

where $W$ is a weight function defined on the computational domain $\Omega$, ranging from zero to unity. This function is used to isolate the region of interest, for example the boundary layer on the top side of a plate (see figure 1).

A Lagrangian $\mathcal{L}$ is defined with the objective of maximizing the perturbation energy at the final time $E(T)$, while satisfying the flow equations and the inflow energy constraint,

$$
\mathcal{L}=E(T)-\frac{1}{T} \int_{0}^{T} \int_{\Omega}\left[\boldsymbol{u}^{\dagger} \cdot(\boldsymbol{N} \boldsymbol{S})+p^{\dagger}(\nabla \cdot \boldsymbol{u})\right] \mathrm{d} \Omega \mathrm{d} t-\lambda\left(\left\|\boldsymbol{u}_{B}^{\prime}\right\|_{b}^{2}-E_{b}\right)
$$

The second term is a constraint that the flow variables satisfy (2.1), and $\boldsymbol{u}^{\dagger}$ and $p^{\dagger}$ are the adjoint velocity and pressure; the last term constrains the $b$-norm of the inflow perturbation to the prescribed value $\sqrt{E_{b}}$, and $\lambda$ is the associated Lagrange multiplier.

A stationary point of the Lagrangian is sought by setting its variation with respect to all independent variables to zero. The gradient of the final energy with respect to the inflow perturbation is then obtained,

$$
\nabla_{\boldsymbol{u}_{B}^{\prime}} E=T^{-1} \int_{0}^{T}\left(p^{\dagger} \boldsymbol{n}-R^{-1} \nabla_{n} \boldsymbol{u}^{\dagger}\right) G \mathrm{~d} t
$$


where $\boldsymbol{n}$ is the unit outward norm on the boundary $\partial \Omega$. The variables $p^{\dagger}$ and $\boldsymbol{u}^{\dagger}$ are solutions to the adjoint equations,

$$
\partial_{t} \boldsymbol{u}^{\dagger}+\boldsymbol{u} \cdot \nabla \boldsymbol{u}^{\dagger}-\nabla \boldsymbol{u} \cdot \boldsymbol{u}^{\dagger}-\nabla p^{\dagger}+R e^{-1} \nabla^{2} \boldsymbol{u}^{\dagger}=0, \quad \nabla \cdot \boldsymbol{u}^{\dagger}=0,
$$

which are integrated backwards from $t=T$ to $t=0$. The adjoint velocity is initialized as $\boldsymbol{u}_{T}^{\dagger}=2 T W \boldsymbol{u}_{T}^{\prime}$. Since the velocity vector $\boldsymbol{u}$ is required in (2.8), it must be saved at every step when integrating the forward equations (2.1).

By iterative integration of the forward-adjoint loop (2.1) and (2.8), the nonlinear optimal inflow perturbation with a specified frequency and energy is obtained (Mao, Blackburn \& Sherwin 2015). This procedure can be repeated for different frequencies and at different magnitudes of the inflow energy, and the boundary-layer response can be contrasted. In the limit of small perturbation energy, the results should be consistent with the outcome of linear analysis. Previous linear optimal perturbation studies have not, however, examined the effect of the leading edge. Therefore, the linearized form of the analysis is also summarized.

\subsection{Linear optimal inflow perturbation}

When the energy of the inflow perturbation and the associated downstream flow response are sufficiently small, the perturbation dynamics can be approximated by the linearized non-dimensional NS equations,

$$
\partial_{t} \boldsymbol{u}^{\prime}+\boldsymbol{U} \cdot \nabla \boldsymbol{u}^{\prime}+\boldsymbol{u}^{\prime} \cdot \nabla \boldsymbol{U}+\nabla p^{\prime}-R e^{-1} \nabla^{2} \boldsymbol{u}^{\prime}=0, \quad \nabla \cdot \boldsymbol{u}^{\prime}=0,
$$

where the left-hand side of the linearized momentum equations will be denoted $\boldsymbol{L N S}$. An energy gain is defined as the ratio of the energy within the domain at the final time to that of the inflow perturbation,

$$
K=\max _{\boldsymbol{u}_{\boldsymbol{B}}^{\prime}} \frac{E(T)}{\left\|\boldsymbol{u}_{\boldsymbol{B}}^{\prime}\right\|_{b}^{2}} .
$$

The gain is thus a normalized measure of the amplification of the inflow perturbation and, due to linearity, is independent of its energy.

The Lagrangian for the linearized system is defined as

$$
\mathcal{L}=K-T^{-1} \int_{0}^{T} \int_{\Omega}\left[\boldsymbol{u}^{\ddagger} \cdot(\boldsymbol{L N S})+p^{\ddagger}\left(\boldsymbol{\nabla} \cdot \boldsymbol{u}^{\prime}\right)\right] \mathrm{d} \Omega \mathrm{d} t-\lambda\left(\left\|\boldsymbol{u}_{B}^{\prime}\right\|_{b}^{2}-E_{b}\right),
$$

and the gradient of $K$ with respect to the inflow perturbation is given by

$$
\nabla_{\boldsymbol{u}_{\boldsymbol{B}}^{\prime}} K=T^{-1} \int_{0}^{T}\left(p^{\ddagger} \boldsymbol{n}-\operatorname{Re}^{-1} \nabla_{n} \boldsymbol{u}^{\ddagger}\right) G \mathrm{~d} t .
$$

The adjoint variables are solutions to the adjoint of the linearized NS equations,

$$
\partial_{t} \boldsymbol{u}^{\ddagger}+\boldsymbol{U} \cdot \nabla \boldsymbol{u}^{\ddagger}-\nabla \boldsymbol{U} \cdot \boldsymbol{u}^{\ddagger}-\nabla p^{\ddagger}+R e^{-1} \nabla^{2} \boldsymbol{u}^{\ddagger}=0, \quad \nabla \cdot \boldsymbol{u}^{\ddagger}=0 .
$$

These equations contain only the base velocity, $\boldsymbol{U}$, and do not require the timedependent forward velocity field $\boldsymbol{u}(t)$. In addition, in the linear limit there is an optimal step size in the optimization procedure (see Mao, Blackburn \& Sherwin 2013, for details). 


\subsection{Secondary instability analysis}

In bypass transition, the receptivity of the boundary layer to free-stream forcing and the amplification of Klebanoff modes are followed by secondary instability of the streaky base profile. Linear theory can be applied to characterize the exponential instabilities that can arise during this stage. In the present study, the base state for the secondary instability analyses will be a cross-flow plane, extracted downstream of the leading edge and comprising the mean flow and the response to the inlet perturbation. Since streaks are of low frequency, the base flow is assumed to be two-dimensional and steady, and the stability analysis in the streamwise direction is parametric. The base state can therefore be denoted $\mathcal{Q}=\left(U_{2}(y, z ; x), V_{2}(y, z ; x), W_{2}(y, z ; x)\right)^{\mathrm{T}}$.

The secondary instability $\boldsymbol{q}_{2}^{\prime}=\left(\boldsymbol{u}_{2}^{\prime}, p_{2}^{\prime}\right)^{\mathrm{T}}$, where $\boldsymbol{u}_{2}^{\prime}=\left(u_{2}^{\prime}, v_{2}^{\prime}, w_{2}^{\prime}\right)$, satisfies the linearized equations,

$$
\partial_{t} \boldsymbol{u}_{2}^{\prime}+\mathcal{Q} \cdot \nabla \boldsymbol{u}_{2}^{\prime}+\boldsymbol{u}_{2}^{\prime} \cdot \nabla \mathcal{Q}+\nabla p_{2}^{\prime}-R e^{-1} \nabla^{2} \boldsymbol{u}_{2}^{\prime}=0 .
$$

A normal-mode assumption is invoked in the streamwise direction,

$$
\boldsymbol{q}_{2}^{\prime}(x, y, z)=\hat{\boldsymbol{q}}_{2}(y, z) \exp (\mathrm{i} \alpha x+\gamma t),
$$

where $\alpha$ is the streamwise wavenumber and $\gamma$ is the temporal growth rate. Substituting this ansatz into the linearized perturbation equations (2.14) yields an eigenvalue problem,

$$
\gamma \hat{\boldsymbol{u}}_{2}=\boldsymbol{A} \hat{\boldsymbol{u}}_{2} .
$$

The eigenspectrum of $\boldsymbol{A}$ describes the modal stability of the underlying base flow $\mathcal{Q}$. We do not construct $\boldsymbol{A}$ explicitly, and instead a spectral approximation of the fundamental solution operator is evaluated using an adaption of the implicitly restarted Arnoldi scheme (Barkley, Blackburn \& Sherwin 2008).

\section{Computational set-up}

The computational domain for flow past a flat plate with a slender leading edge is depicted in figure 1(a). The domain spans from $x=-20$ to $x=200$ in the streamwise direction, and from $y=-40$ to $y=40$ in the vertical direction. For three-dimensional computations, a spectral decomposition into spanwise wavenumbers, $\beta$, is adopted with 64 Fourier modes over the width of the domain $L_{z}=12$. The leading edge of the plate is elliptic, with semi-major axis $L$ and semi-minor axis $R$, and aspect ratio $A R=L / R=20$. The half-thickness of the plate $R$ (or $D / 2$ ) and the free-stream speed $U_{\infty}$ are used as the reference length and velocity, respectively. This 'full' domain is adopted for nonlinear computations, while the linear analyses consider the top surface of the plate only, with the option of symmetric $(\partial u / \partial n=0, \quad v=0, \quad \partial w / \partial n=0)$ or antisymmetric $(u=0, \quad \partial v / \partial n=0, \quad w=0)$ boundary conditions at $y=0$ (figure $1 b$ ). This simplification for the linear study was adopted in order to reduce the computational cost in favour of exploring a large range of the parameter space.

A spectral element method is used to discretize the governing equations (Karniadakis $\&$ Sherwin 2005). The computational domain in figure 1(a) is decomposed into 2843 elements, clustered around the upper plate. The mesh for figure $1(b)$ is the same as the upper part of figure 1(a), and consists of 1824 spectral elements. Each element is further decomposed into a $(P+1) \times(P+1)$ grid using a spectral method, where $P$ represents a polynomial order and can be used to refine the resolution (Karniadakis \& Sherwin 2005). In this work, $P=7$ was adopted in order to ensure that the results are independent of resolution. 

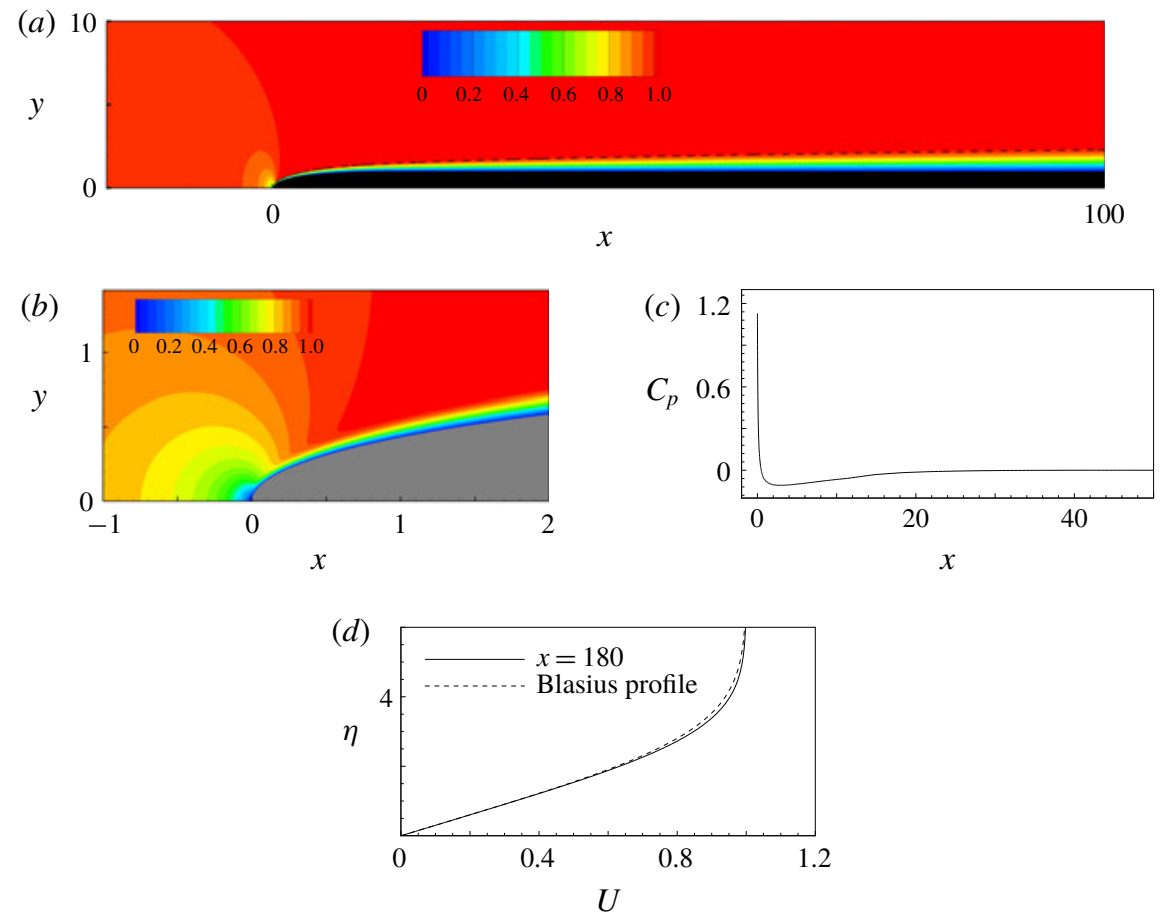

FIgURE 2. (Colour online) The base flow at $R e=800$. $(a, b)$ Contours of streamwise velocity around the upper part of the full domain and the leading edge, respectively. (c) The pressure coefficient along the surface of the plate. $(d)$ Comparison of the velocity profile at $x=180$ with the Blasius solution. The dashed line in $(a)$ marks the $99 \%$ boundary-layer thickness, $\delta_{99}$.

A steady two-dimensional state is first evaluated and used as the base state for the perturbation analyses. Since the flow is globally stable, the base flow is computed by marching in time the full nonlinear NS equations (2.1) to a steady solution. The base flow profile at $R e=800$ is illustrated in figure 2. The development of a boundary layer over the leading edge and the following plate can be seen from figure $2(a, b)$. Around the leading edge, the flow is subjected to a favourable and subsequently an adverse pressure gradient (see figure $2 c$ ). However, on the flat-plate region downstream, the flow develops into a canonical zero-pressure-gradient boundary layer. The comparison of the downstream velocity profile at $x=180$ with the Blasius solution is shown in figure $2(d)$.

\section{Linear perturbation analysis}

We first examine the gain of inflow perturbations in the linear framework where the magnitude of the disturbances is assumed to be infinitesimal. As noted above, the half-domain shown in figure $1(b)$ is adopted. As the flat plate is two-dimensional, the inflow perturbation and its response are expressed in terms of a Fourier expansion in the spanwise direction,

$$
\boldsymbol{u}_{\boldsymbol{B}}^{\prime}(y, z)=\sum_{\beta=0}^{\infty} \boldsymbol{u}_{\boldsymbol{B}, \beta}^{\prime}(y) \mathrm{e}^{\mathrm{i} \beta z} \quad \text { and } \quad \boldsymbol{u}^{\prime}(x, y, z)=\sum_{\beta=0}^{\infty} \boldsymbol{u}_{\beta}^{\prime}(x, y) \mathrm{e}^{\mathrm{i} \beta z},
$$




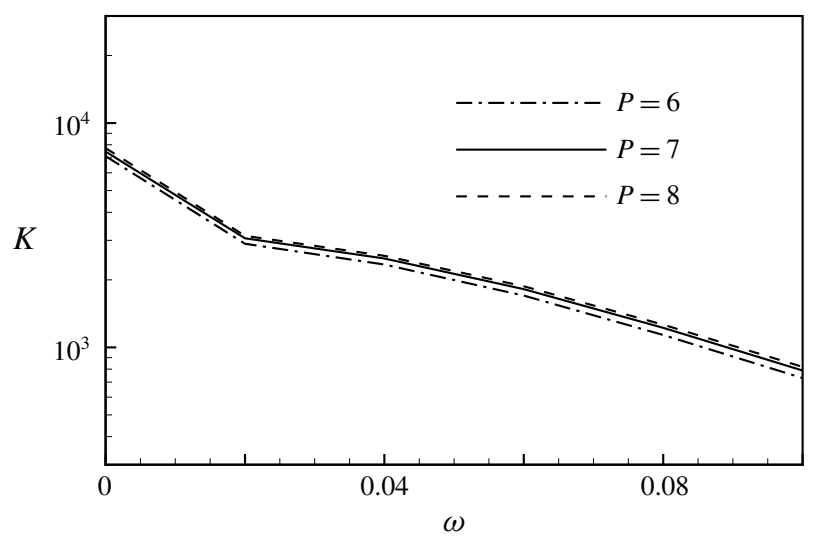

FIGURE 3. Convergence of the gain $K$ of symmetric linear inflow perturbations with respect to $P$ at $R e=800, \beta=1.4$ and various $\omega$.
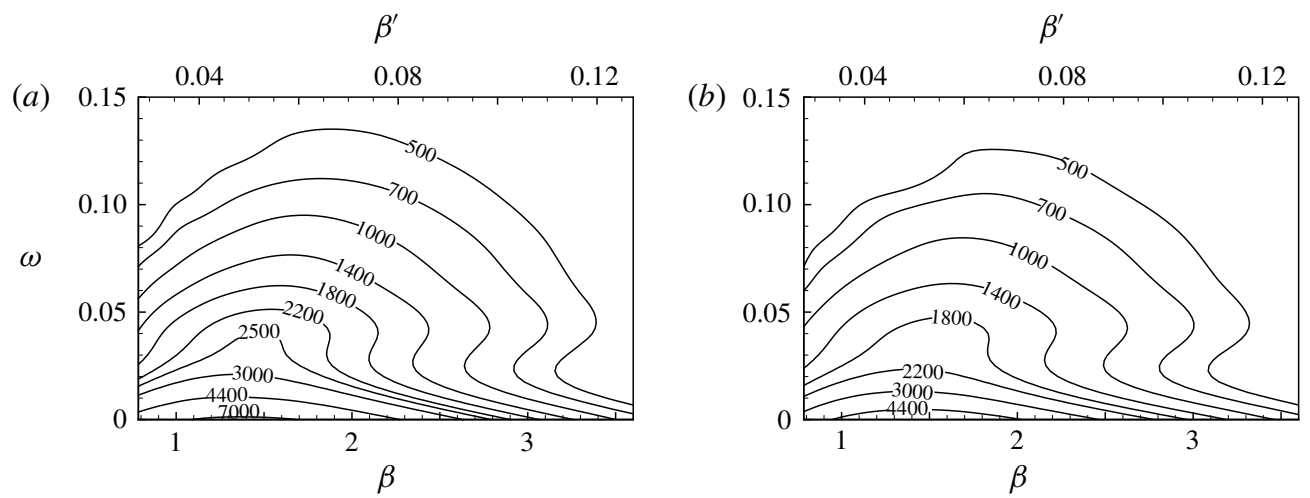

FIgURE 4. Contour of the gain $K$ for linear optimal inflow perturbations at $R e=800$ with (a) symmetric and $(b)$ antisymmetric boundary conditions at $y=0$. Symmetric conditions will be used in all the following linear studies.

where $\beta$ denotes the spanwise wavenumber. In the linear limit, waves with different $\beta$ are decoupled. Therefore, each linear calculation is essentially two-dimensional, but with a prescribed spanwise wavenumber.

To demonstrate the convergence of the numerical method when solving the perturbation equations, figure 3 shows the maximum linear gain $K$ for symmetric inflow perturbations as a function of the inflow frequency $\omega$, when different polynomial orders are used, $P=\{6,7,8\}$. These results were obtained at $R e=800$, which is the largest Reynolds number considered in this work, and at spanwise wavenumber $\beta=1.4$. The results show that a value of $P=7$ is sufficient to ensure convergence.

The gains due to symmetric and antisymmetric inflow perturbations are presented in figure 4 over a range of frequencies and spanwise wavenumbers. In both cases, the most energetic flow response is associated with steady inflow perturbations, $\omega=0$, and at $\beta=1.4$. At higher frequencies, the maximum gain is obtained at higher spanwise wavenumbers. Over the range of parameters considered, the symmetric perturbations 

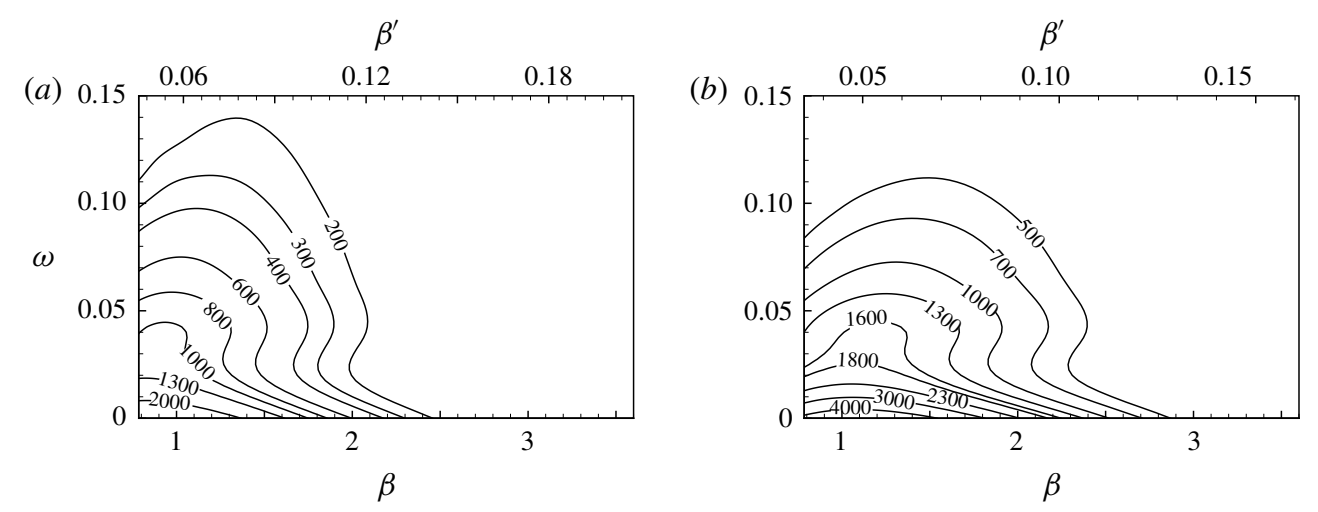

FIGURE 5. Contour of the gain $K$ of linear optimal inflow perturbations at $(a) \operatorname{Re}=300$ and (b) $\operatorname{Re}=500$.

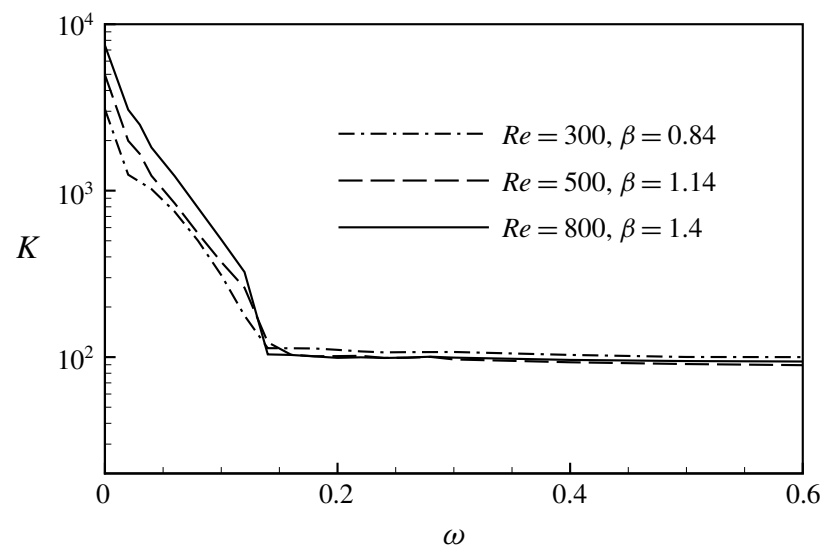

FIGURE 6. Variation of the gain with $\omega$ at optimal combinations of $R e$ and $\beta$.

result in larger energy growth than antisymmetric ones, and hence will be the focus of the remainder of the linear analyses.

The effect of Reynolds number on the gain is shown in figures $5(a, b)$, which should also be compared to figure $4(b)$. As $R e$ is increased from 300 to 500 and finally 800 , the flow response to the inlet perturbation becomes more energetic. The maximum response remains at $\omega=0$, whereas the spanwise wavenumber increases from $\beta=0.84$ to 1.14 and 1.4. Note that the present spanwise wavenumber is non-dimensionalized by the half-thickness of the flat plate. If, alternatively, the normalization $\beta^{\prime} \equiv \beta / \sqrt{R e}$ (Luchini 2000; Andersson et al. 2001) is adopted, the optimal wavenumbers become $\beta=\{4.85,5.10,4.95\} \times 10^{-2}$. Therefore, appropriately scaled, the optimal spanwise wavenumber becomes insensitive to the Reynolds number for the range of parameters examined herein (see the upper axis in figures 4 and 5).

For each Reynolds number considered above, the gain is plotted against the frequency at the optimal spanwise wavenumber in figure 6. As $\omega$ increases, the gain reduces monotonically until $\omega \approx 0.14$ where $K$ becomes nearly constant. Therefore $\omega \approx 0.14$ can be considered as a cutoff frequency. The higher frequencies are thus 

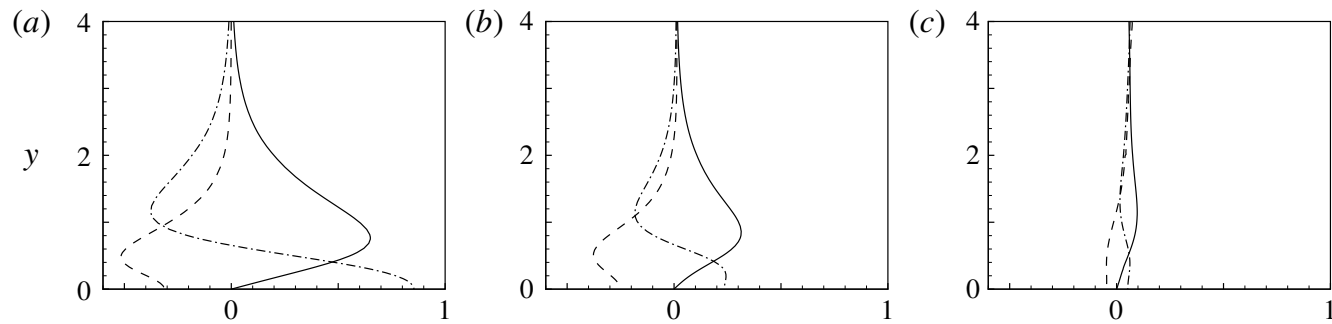

FIGURE 7. Velocity profile of optimal inflow perturbations at $\beta=1.4$ and $R e=800$ : perturbation components for $(a) \omega=0,(b) \omega=0.12$ and (c) $\omega=0.24$; with - - - for $u$, for $v$ and $-\cdot-\cdot-$ for $w$.
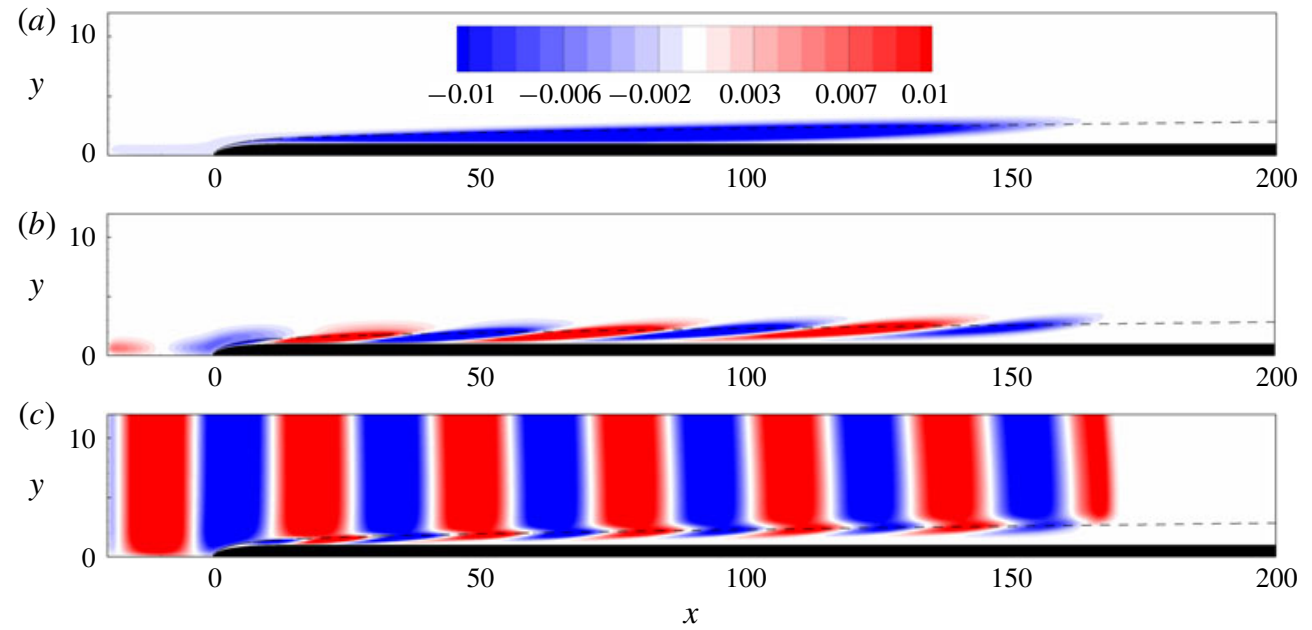

FIGURE 8. (Colour online) Side views of isosurfaces of streamwise perturbation velocity at $\operatorname{Re}=800, T=180$ and $(a) \omega=0,(b) \omega=0.12$ and $(c) \omega=0.24$. The same contour levels are adopted in panels $(a-c)$. The dashed line marks the boundary-layer $99 \%$ thickness.

relatively ineffective at perturbing the boundary layer. The associated perturbations do not penetrate the boundary layer, and are simply advected in the free stream.

The velocity profiles of the linearly optimal inflow perturbations are presented in figure 7. At frequencies below the cutoff value (figure $7 a, b$ ), the perturbation is concentrated around $y=0$, and thus impinges onto the leading edge and interacts with the boundary layer. At frequencies higher than the cutoff value (figure $7 c$ ), the perturbation is approximately uniform in the cross-flow plane at the inlet.

Applying the linear optimal perturbations at the inflow and integrating the linearized equations (2.9), we compute the linear flow response. The Reynolds number is $R e=800$, the spanwise wavenumber is $\beta=1.4$, and three frequencies are considered, $\omega=0, \omega=0.12$ and $\omega=0.24$. A side view of the boundary-layer response at $T=180$ is shown in figure 8 . As noted above, the perturbations with frequencies below the cutoff value $\omega \approx 0.14$ can penetrate the boundary layer (see figure $8 a, b$ ), while the high-frequency perturbations only reside in the free stream (figure $8 c$ ).

The cutoff frequency in figure 6 is a manifestation of shear sheltering. Free-stream disturbances with low streamwise wavenumbers and optimal profiles in the cross-flow 

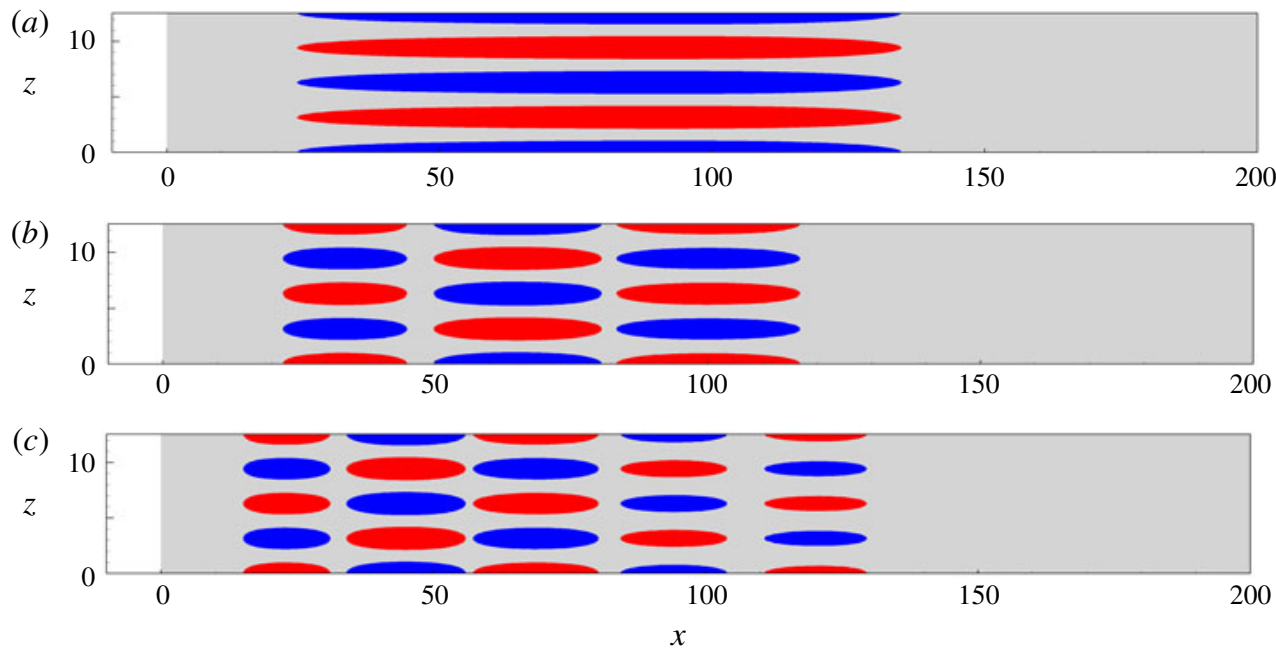

FIGURE 9. (Colour online) Top views of isosurfaces of streamwise perturbation velocity 0.02 (red) and -0.02 (blue) at $\operatorname{Re}=800, \beta=1.4$ and $(a) \omega=0,(b) \omega=0.08$ and (c) $\omega=0.12$. The grey colour represents the flat plate. The inflow perturbation is optimal and has unit energy.

plane can penetrate into the boundary layer and generate a strong distortion due to the absence of a restoring pressure, while those with high wavenumbers are filtered by the shear (Hunt \& Durbin 1999; Zaki \& Saha 2009). The theory of shear sheltering captures the dependence of the penetration depth of external disturbances into the boundary layer, $d_{p}$, measured from the edge of the boundary layer towards the wall. This depth is proportional to the fluid viscosity $(v)$ and the square of the wall-normal wavenumber $\left(k_{y}^{2}\right)$, and is inversely proportional to the shear rate $(\dot{\gamma})$ and streamwise wavenumber $\left(k_{x}\right)$, such that $d_{p} \propto\left(\nu k_{y}^{2} / \dot{\gamma} k_{x}\right)$. The denominator captures that strong shear filters the free-stream perturbations, unless they are very long in the streamwise direction. The present results are consistent with this theory, with the shielded disturbance approximately uniform in the vertical direction in the free stream, corresponding to a zero vertical wavenumber (see figure $8 c$ ). It is worth noting that in the wall-normal direction, the theory predicts an opposite trend with large scales expelled and small scales penetrating the shear (Zaki \& Saha 2009).

Since disturbances with frequencies above the cutoff value in figure 6 do not induce a boundary-layer response, they are not considered further. Instead, we place our attention on the downstream linear evolution of inflow perturbations with frequencies below the cutoff value. Figure 9 compares the boundary-layer response at three frequencies, $\omega=\{0,0.08,0.12\}$ at the time when the response is most energetic. Only the streamwise perturbation velocity, which is the dominant component, is shown. At $\omega=0$, the inflow perturbation induces elongated, high-amplitude velocity streaks inside the boundary layer, which have been studied extensively in connection with bypass transition to turbulence. At higher values of $\omega$, the streaks become shorter and weaker. This observation reinforces the importance of the low-frequency components of free-stream turbulence in the early stages of transition, even when the leading edge is taken into consideration.

The target (or final) time $T$ is then varied to examine its influence on the optimal development, as shown in figure 10. At higher $T$, the flow response is 

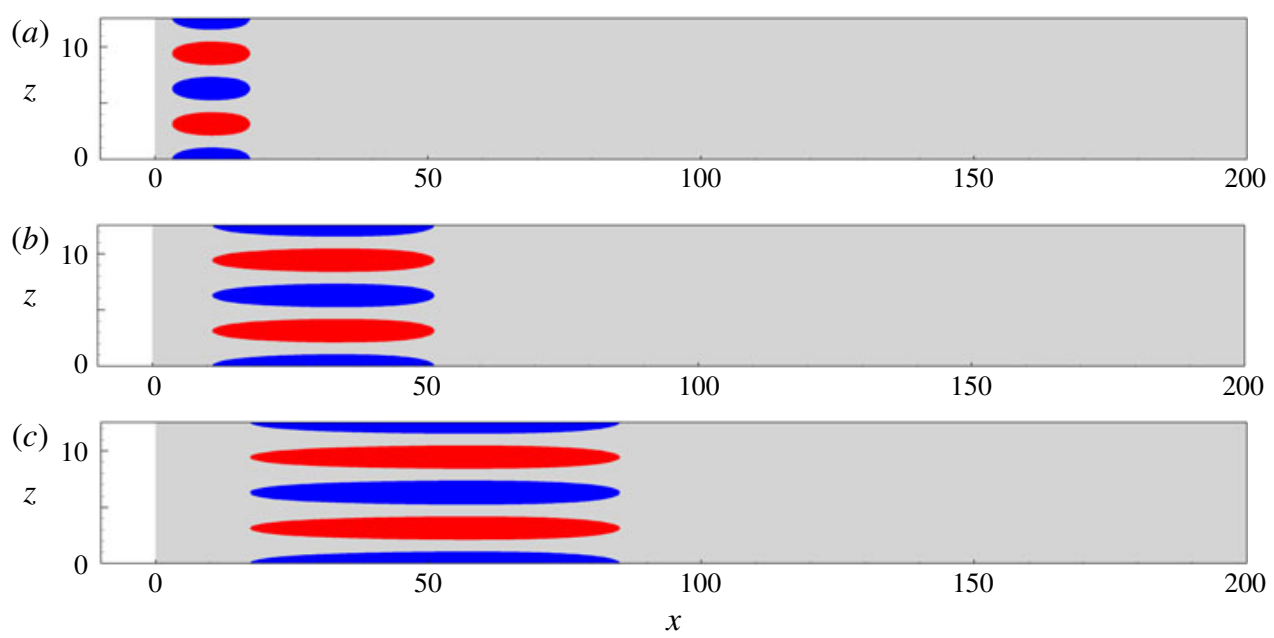

FIgURE 10. (Colour online) Top views of isosurfaces of streamwise perturbation velocity 0.02 (red) and -0.02 (blue) at $R e=800, \beta=1.4$, and $(a) T=40,(b) T=80$ and (c) $T=120$. The grey colour represents the flat plate. The inflow perturbation is optimal and has unit energy.

more pronounced and expands towards the outflow. Over the range of parameters considered, the boundary-layer response remains qualitatively unchanged and has the form of elongated streamwise streaks.

\section{Nonlinear perturbation analysis}

When the energy of the inflow disturbance is sufficiently large, the linear assumption does not hold and the nonlinear algorithms are adopted to study the perturbation dynamics. The inflow perturbation is restricted to be monochromatic in time in order to isolate the contribution of each frequency. As such, the temporal decomposition of the inflow perturbation in (2.3) is still adopted, and the generation of higher temporal harmonics by nonlinearity takes place downstream. Since all spanwise modes are coupled in the nonlinear case, the full three-dimensional problem is solved and the shape of the inlet disturbance includes a superposition of waves. In addition, the symmetric and antisymmetric perturbations are coupled and, as a result, both the upper and lower surfaces of the plate are simulated although the grid resolution is concentrated on the upper half. In order to isolate the top surface, $W$ is set to unity for $y \geqslant 0$ and reduces exponentially to zero in the lower half of the domain. When the energy of the inflow disturbance is restricted to a small magnitude, it is anticipated that the outcome of the nonlinear computations converges to the linear results from $\S 4$. In this limit, the inflow perturbation should feature a single spanwise wavenumber.

The Reynolds number is $R e=800$ and the focus is directed to the case with $\omega=0$, at which the inflow perturbation is most amplified in the linear limit. Four energy levels, $\left\|\boldsymbol{u}_{B}^{\prime}\right\|_{b}=\{0.5,1,2,3\} \times 10^{-2}$, are considered. The resulting nonlinear optimal inflow perturbations are reported in figure 11 . The contour levels have been scaled by the energy of the inflow disturbance in order to facilitate comparison. The inflow perturbation consists of streamwise ( $x$ direction) and vertical ( $y$ direction) vorticity components and, for all the cases studied, the former has a larger magnitude. 

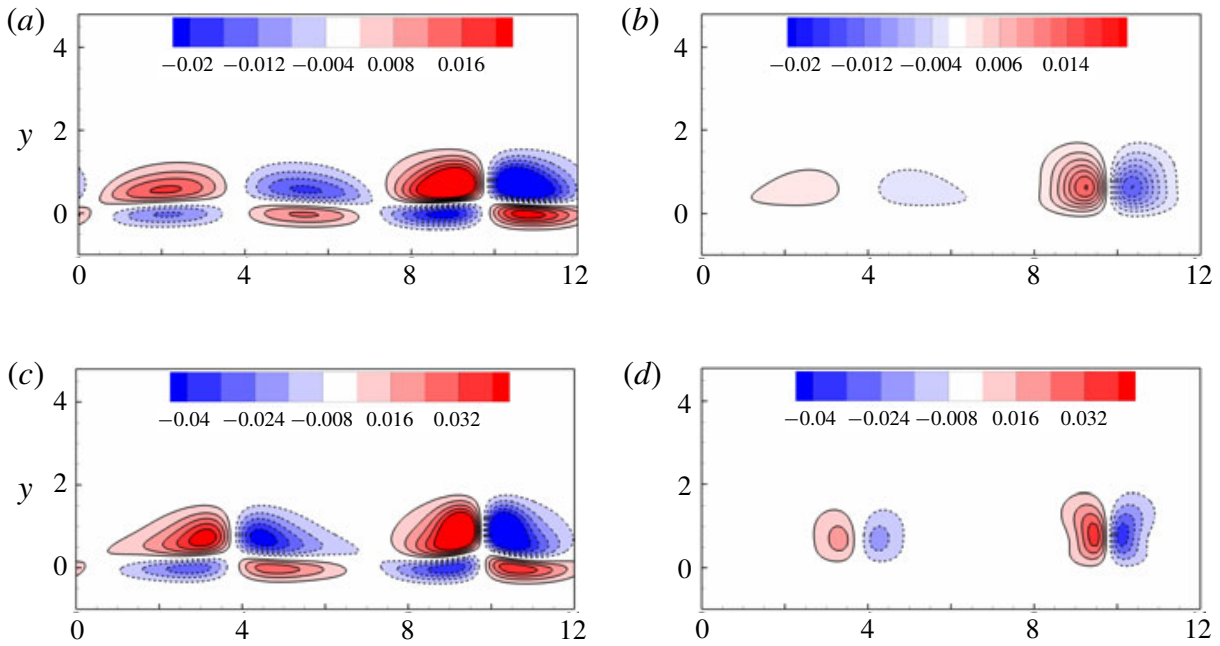

(d)
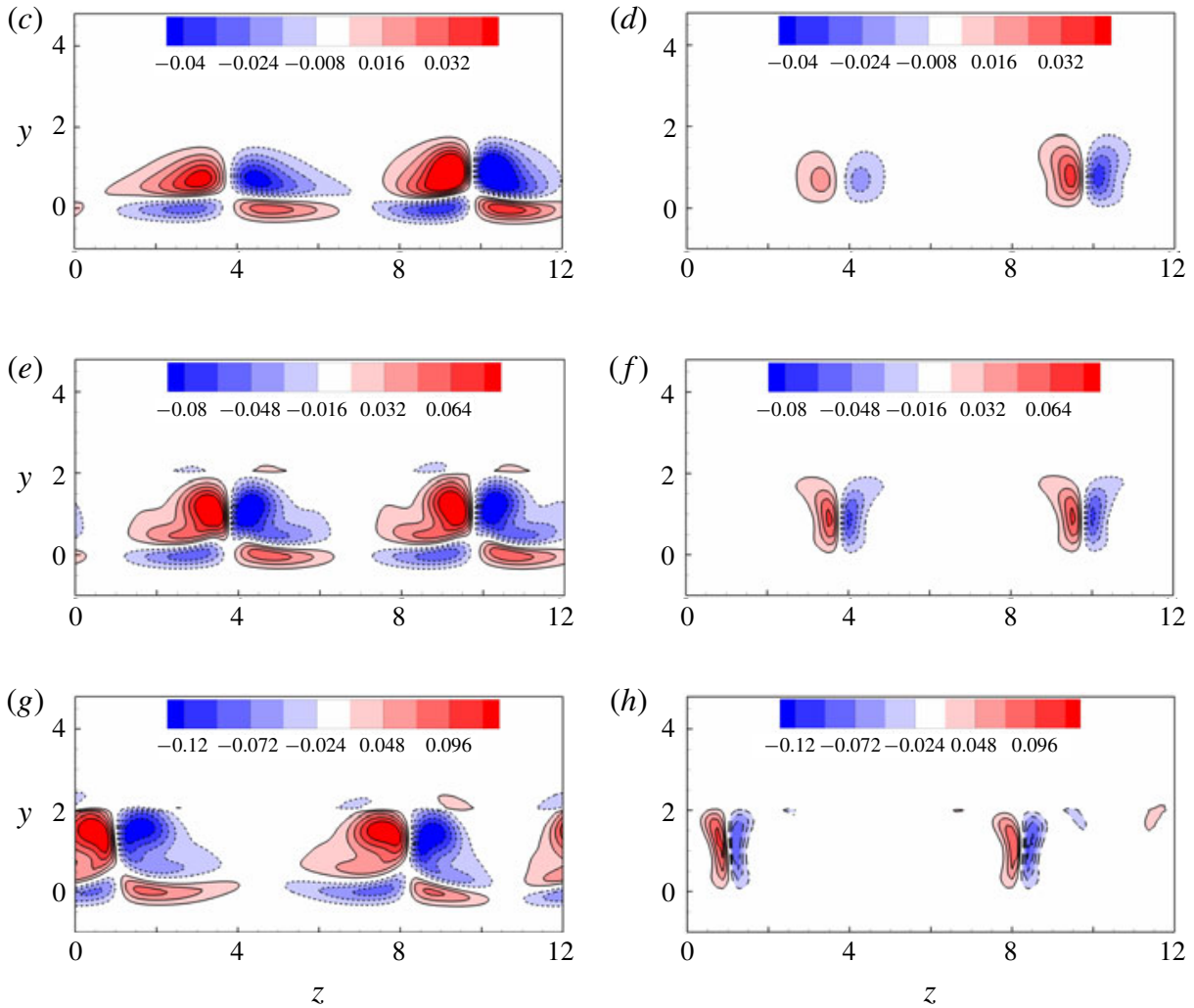

FIGURE 11. (Colour online) Nonlinear optimal inflow perturbations at $R e=800, T=$ 180, $\omega=0$ and $\left\|\boldsymbol{u}_{B}^{\prime}\right\|_{b}=0.5 \times 10^{-2}, 10^{-2}, 2 \times 10^{-2}$ and $3 \times 10^{-2}$ from top to bottom. The left and right columns are the streamwise ( $x$ direction) and vertical ( $y$ direction) vorticity, respectively. Solid and dashed lines represent positive and negative contour levels, respectively.

At higher energy of the inflow perturbation, both vorticity components become increasingly more localized and their peaks shift away from $y=0$ towards $y \approx 1$, which is near the edge of the downstream boundary layer.

The responses to the inflow disturbances are shown in figure 12. Low- and highspeed velocity streaks amplify inside the boundary layer, at similar heights within the boundary layer when the energy of the inflow perturbation is small. When the inflow disturbance is more energetic, the low-speed streaks are lifted away from the surface while the high-speed ones are shifted towards the wall. In an earlier study, this effect 
(a)

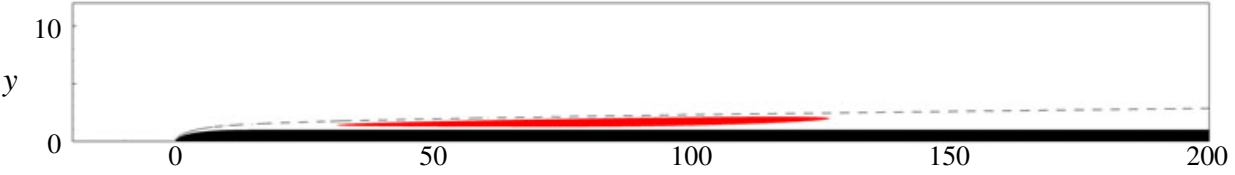

(b)

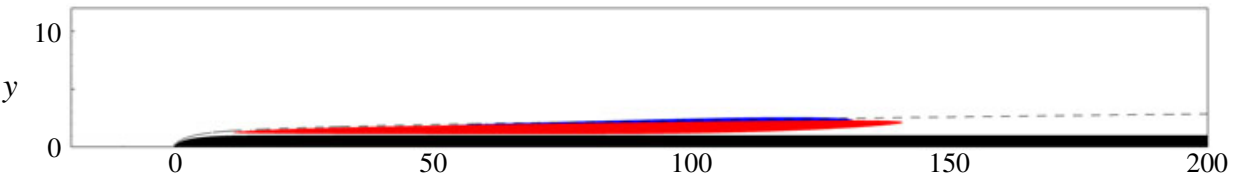

(c)

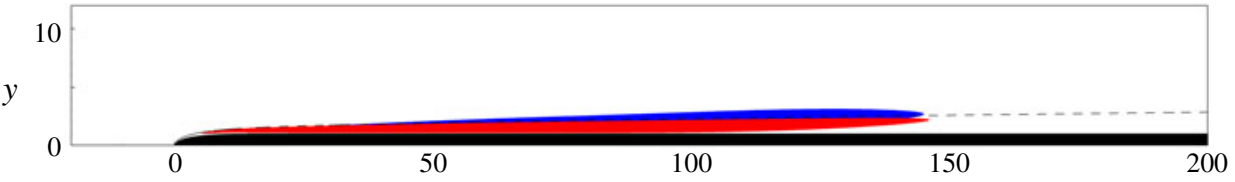

(d)

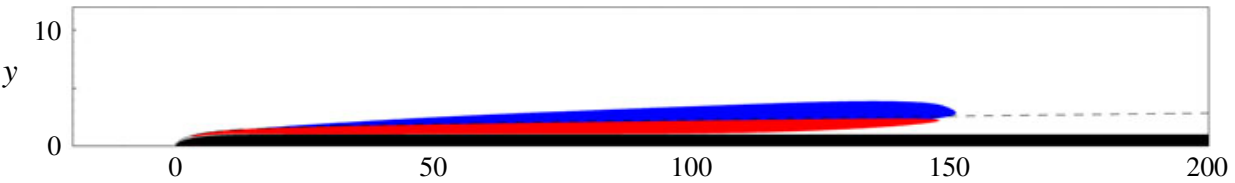

(e)

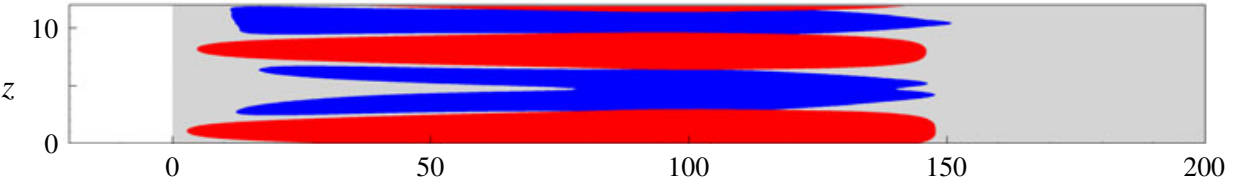

$(f)$

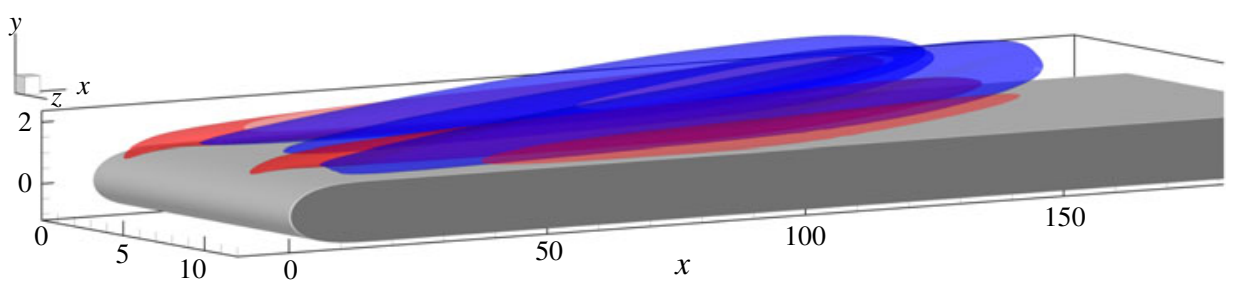

FIGURE 12. (Colour online) Side views of isosurfaces of streamwise perturbation velocity 0.1 (red) and -0.1 (blue) at $R e=800, T=180, \omega=0$ and $\left\|\boldsymbol{u}_{B}^{\prime}\right\|_{b}=0.5 \times 10^{-2}, 10^{-2}$, $2 \times 10^{-2}$ and $3 \times 10^{-2}$ from $(a)$ to $(d)$. The dashed line marks the boundary-layer $99 \%$ thickness. Panels $(e)$ and $(f)$ are top and three-dimensional views of $(d)$, respectively.

was explained in terms of a nonlinear lift-up mechanism, and it was shown to render the flow unstable to high-frequency perturbations (Mao et al. 2017).

In order to illustrate how the inflow streamwise and vertical vorticity induce the streaky boundary-layer response, we examine the leading-edge region. Since the mean flow turns around the leading edge, we consider the components of the perturbation field that are tangent and normal to the base state, $\omega_{t}^{\prime} \equiv \boldsymbol{\omega}^{\prime} \cdot \hat{\boldsymbol{e}}_{t}$ and $\omega_{n}^{\prime} \equiv \boldsymbol{\omega}^{\prime} \cdot \hat{\boldsymbol{e}}_{n}$, where $\hat{\boldsymbol{e}}_{t} \equiv(U, V) /|\boldsymbol{U}|$ and $\hat{\boldsymbol{e}}_{n}=(V,-U) /|\boldsymbol{U}|$. The associated spanwise-averaged squared 


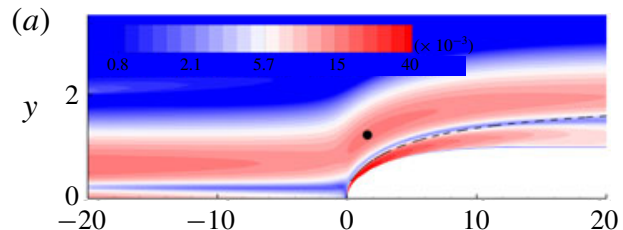

(b)
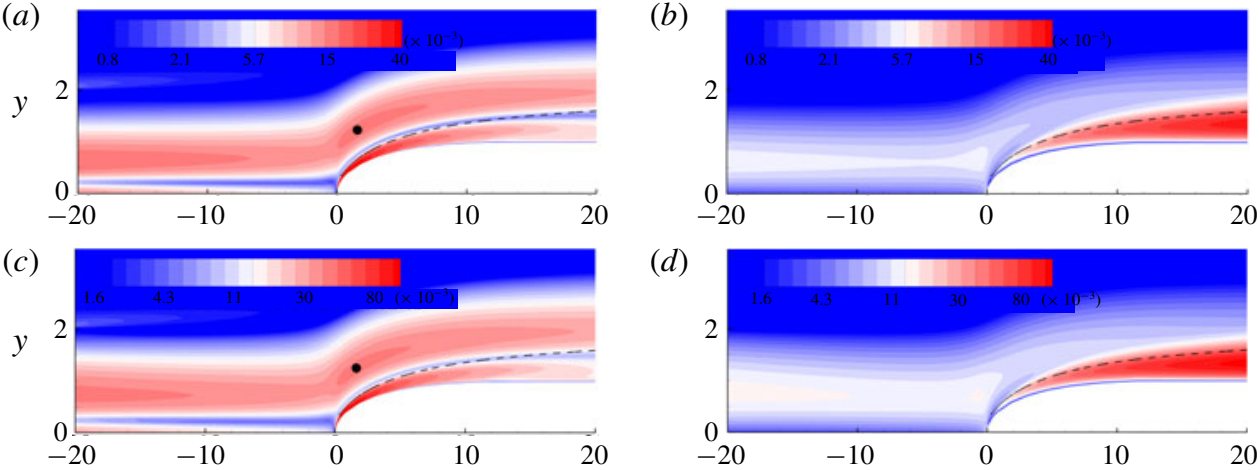

(d)
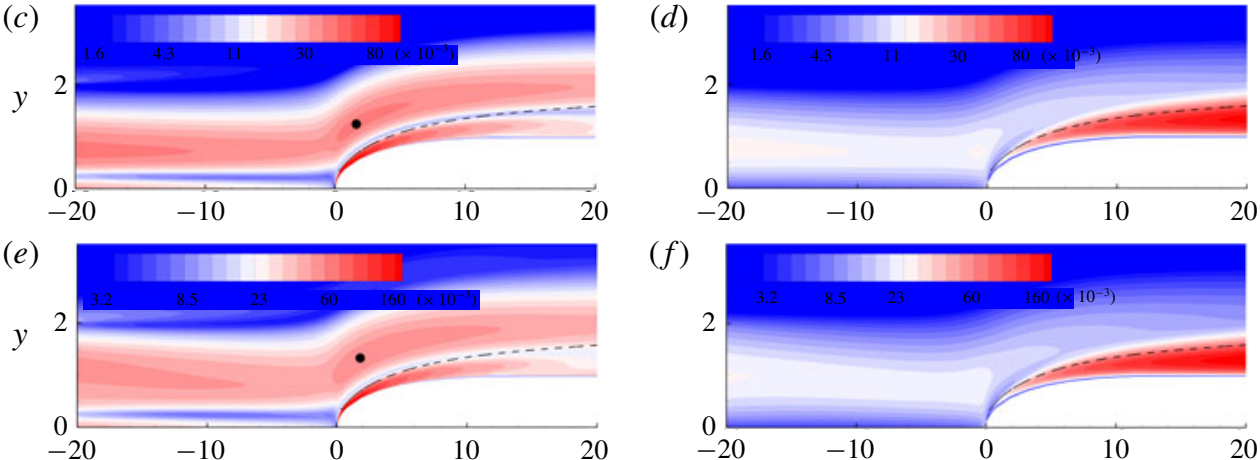

(f)
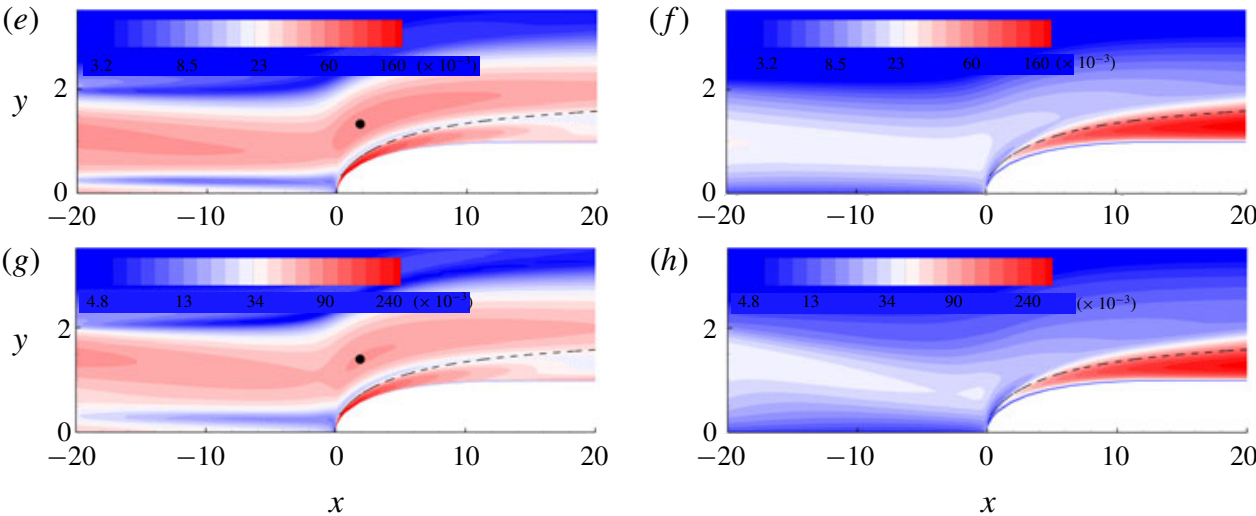

(h)

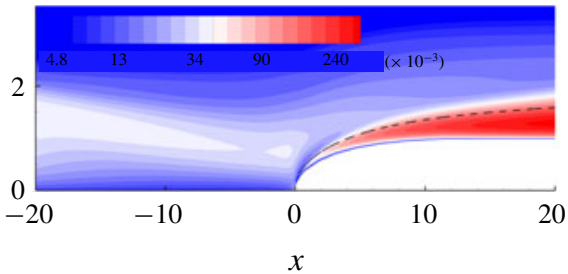

Figure 13. (Colour online) Contours of $(a, c, e, g) \mathcal{E}_{\omega_{t}^{\prime}}$ and $(b, d, f, h) \mathcal{E}_{\omega_{n}^{\prime}}$ at $T=160$ in response to the optimal inflow at $R e=800, \omega=0$ and $\left\|\boldsymbol{u}_{B}^{\prime}\right\|_{b}=0.5 \times 10^{-2}, 10^{-2}, 2 \times$ $10^{-2}$ and $3 \times 10^{-2}$ from top to bottom. The black dots highlight the local maximum of the streamwise vorticity on the left and the dashed lines mark the boundary-layer $99 \%$ thickness. Contour levels are in logarithmic scale.

vorticities are

$$
\mathcal{E}_{\omega_{t}^{\prime}}=\frac{1}{L_{z}} \int_{0}^{L_{z}} \omega_{t}^{\prime 2} \mathrm{~d} z \quad \text { and } \quad \mathcal{E}_{\omega_{n}^{\prime}}=\frac{1}{L_{z}} \int_{0}^{L_{z}} \omega_{n}^{\prime 2} \mathrm{~d} z
$$

Contours of $\mathcal{E}_{\omega_{t}^{\prime}}$ and $\mathcal{E}_{\omega_{n}^{\prime}}$ starting at the inflow plane through the leading-edge region are shown in figure 13 . Here $T=160$ is used, rather than the target time $T=180$, so the inflow disturbance is also visible (cf. (2.3)). Similarly as in figure 11, the contour levels are normalized by the energy of the inflow disturbance to facilitate their comparison. Local maxima of $\mathcal{E}_{\omega_{t}^{\prime}}$ near the edge of the boundary layer are marked by black dots. When the free-stream perturbation is advected around the leading edge, it is stretched and tilted by the curved mean-flow streamlines (Leib, Wundrow \& Goldstein 1999). This effect can be expected to be more prominent for a blunt geometry as noted by Schrader et al. (2010). Downstream, over the flat-plate region, the streamwise vorticity itself is decaying; However, it also tilts the mean shear to generate an energetic streak response within the boundary layer, in particular near the upper part, via the lift-up mechanism. 


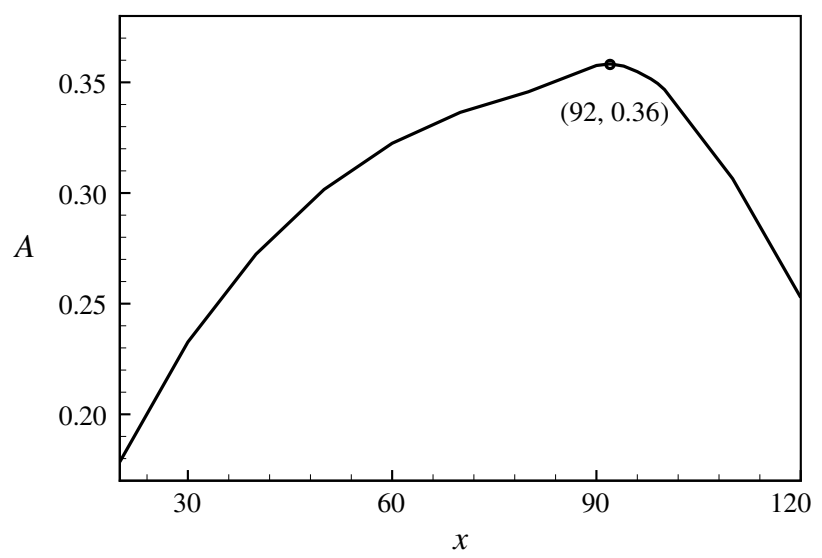

FIgURE 14. Variation of streak amplitude $A$ with streamwise location $x$ at $R e=800, \omega=0$, $T=180$ and $\left\|\boldsymbol{u}_{B}^{\prime}\right\|_{b}=2 \times 10^{-2}$.

The evolution of the inflow perturbation is thus initially dominated by the leadingedge effect, which tilts the perturbation vorticity into the streamwise component. Subsequently, immediately downstream of the leading edge, linear lift-up amplification of boundary-layer streaks takes place in a manner qualitatively similar to the results in figure 10. The linear stage is followed by nonlinear effects, where low-speed streaks breach towards the free stream and high-speed ones are displaced towards the wall (figure 12).

\section{Secondary instability of streaks and transition to turbulence}

\subsection{Secondary instability analysis}

At sufficiently large energy of the inflow perturbation, the boundary-layer response becomes nonlinear: both the negative and positive perturbations reach high amplitudes, with the former being displaced towards the free stream and the latter towards the wall (see figure 12). This configuration is prone to secondary instabilities, in particular when it is exposed to high-frequency forcing, for example due to free-stream turbulence. The secondary instabilities of the streaky boundary layer are herein examined using biglobal stability analysis. The base flow is a cross-flow plane extracted from the full nonlinear NS computations of the boundary-layer response to the optimal inflow forcing. The Reynolds number is $R e=800$, and the inflow parameters are $\omega=0$ and $\left\|\boldsymbol{u}_{B}^{\prime}\right\|_{b}=2 \times 10^{-2}$. At $T=180$, the cross-flow planes are extracted at various streamwise locations and their temporal stability eigenspectra are evaluated.

In previous studies, the stability of streaky boundary layers was correlated with the streak amplitude (e.g. Cossu \& Brandt 2002; Biancofiore, Brandt \& Zaki 2017),

$$
A=\frac{1}{2}\left[\max \left(U-U_{b}\right)-\min \left(U-U_{b}\right)\right],
$$

where $U_{b}$ is the streamwise velocity of the base flow. The streak amplitude in the present study first increases with $x$ then decreases, as shown in figure 14. The maximum amplitude is $36 \%$, and located at $x=92$. It is important to note that, in addition to the amplitude of the streaks being destabilizing, their shape, or functional 

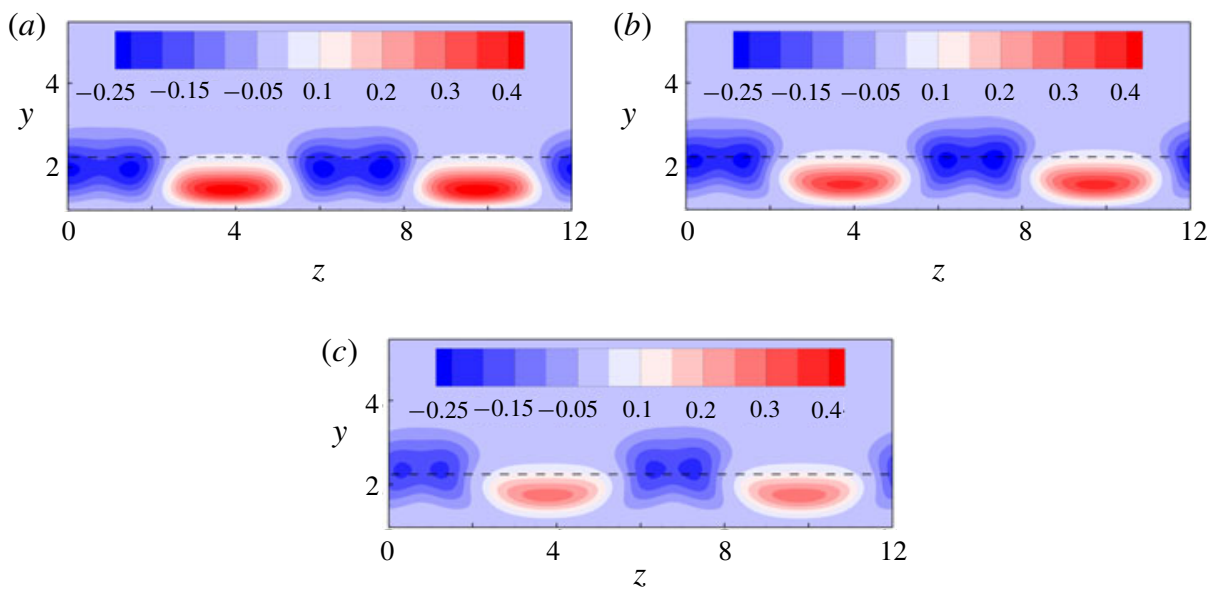

FIGURE 15. (Colour online) Streak perturbations at various streamwise locations: $(a) x=$ 92, (b) $x=106$ and (c) $x=120$. The dashed lines mark the boundary-layer $99 \%$ thickness.

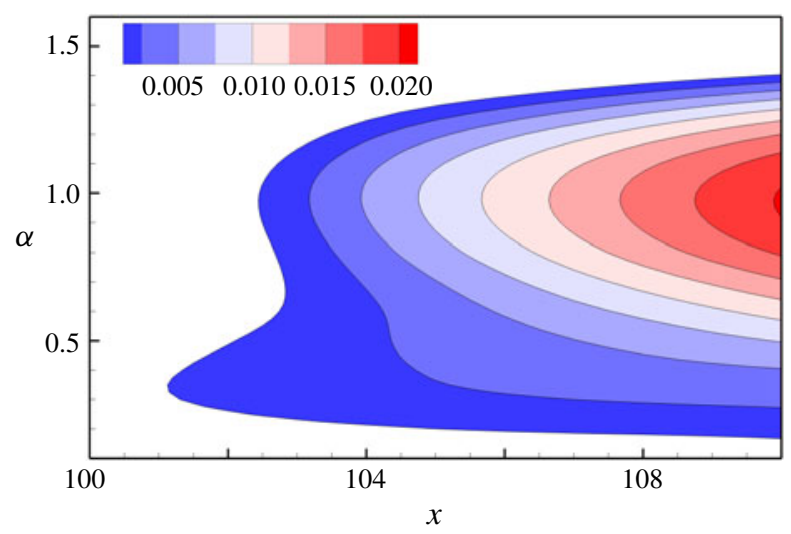

FIGURE 16. (Colour online) The secondary instability growth rate $\gamma$ in the $\alpha-x$ plane for $R e=800, \omega=0, T=180$ and $\left\|\boldsymbol{u}_{B}^{\prime}\right\|_{b}=2 \times 10^{-2}$.

form, is also important (Andersson et al. 2001). In addition, Vaughan \& Zaki (2011) later demonstrated that two general classes of streak instabilities are possible, and termed them 'outer' and 'inner' modes depending on the height of their critical layers and their respective phase speeds.

The secondary instability of the streaky base state was evaluated at various downstream locations in the region from $x=92$ to $x=106$; the perturbation streaks at these locations are plotted in figure 15. In this region the boundary-layer thickness is approximately $1.35 R$. At every position, the streamwise wavenumber $\alpha$ was varied, and the growth rates of the associated most unstable modes are plotted in figure 16 . The onset of instability, or positive growth rate of the temporal modes, appears at $\alpha=0.35$ and $x \approx 101$, where the amplitude of the streaks reaches $35 \%$ of the free-stream speed. At larger $x$, the base flow becomes more unstable even though the streak amplitude, as defined above in (6.1) and illustrated in figure 15, is slightly reduced. This further destabilization is therefore associated with the change in the 

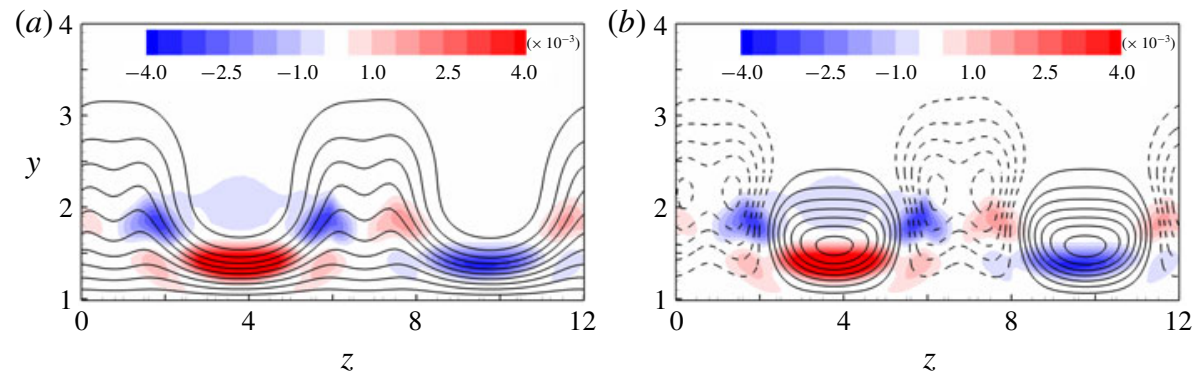

FIgURE 17. (Colour online) Secondary instability modes at $x=106$ and $\alpha=1$. The black lines in $(a)$ are contour lines of streamwise base velocity from 0.1 to 1 and in $(b)$ are contour lines of streaks from -0.25 to 0.35 . The colour contours denote streamwise perturbation velocity.

streak shape, which becomes non-periodic in the spanwise direction due to the nonlinear lift-up (Mao et al. 2017). The fastest-amplifying instability has a streamwise wavenumber $\alpha \approx 1$. At higher inflow perturbation levels, e.g. $\left\|\boldsymbol{u}_{B}^{\prime}\right\|_{b}=3 \times 10^{-2}$ (not shown here), the region for secondary instability moves upstream and the most unstable streamwise wavenumber reduces.

A representative eigenmode of the secondary instability at the optimal streamwise wavenumber is illustrated in figure 17, along with line contours of the streaky base state. The mode is symmetric with respect to the high-speed streaks. It is concentrated in the near-wall region, and can be regarded as an inner secondary instability according to the classification by Vaughan \& Zaki (2011). The streaky base state may also have an outer instability, although with lower growth rate than the most unstable mode in figure 17 . Which secondary instability emerges in simulations of transition depends on the growth rate and also the initial seed, or receptivity.

\subsection{Transition to turbulence}

As discussed in the sections on the nonlinear perturbation and secondary instability, a low-frequency inflow disturbance is required to generate the streaks, which will become unstable to high-frequency perturbations. In this section, we examine the breakup of streaks and the transition to turbulence, by performing DNS of the steady inflow optimal perturbation supplemented with high-frequency body forcing to activate the secondary instability. The forcing is added upstream of the secondary instability region, from $x=88$ to $x=92$, and is concentrated in the boundary layer. The flow development over the flat plate is shown in figure 18. The streamwise velocity at $z=2$ is plotted in figure 18(a) where, owing to the choice of contour levels, the inflow disturbance is not visible. A three-dimensional view of the streamwise perturbation is shown in figure $18(b)$, where the streaks, their secondary instability and breakdown to turbulence are clearly captured.

The entire transition process, starting from the free-stream perturbation, can be described in the following manner. Upstream of a slender leading edge, the most effective forms of linear and nonlinear perturbations are steady. This result is due to two effects where low frequencies are favoured: (i) low-frequency perturbations are the most effective at penetrating the shear, even in nonlinear computations with a leading edge; and (ii) the nonlinear amplification of streaks is most pronounced for streamwise structures. Had either mechanism favoured oblique modes, perhaps 
(a)

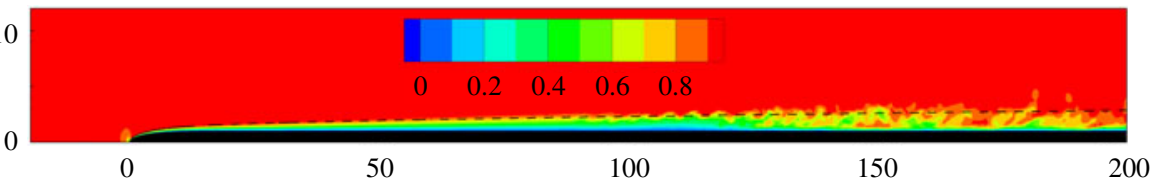

(b)

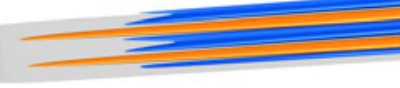

FIgURE 18. (Colour online) Visualization from DNS of transition at $R e=800$. (a) Contours of streamwise velocity at $z=2$. The dashed line marks the boundary-layer $99 \%$ thickness. (b) Isosurfaces of streamwise perturbation velocity with magnitude 0.1 (red) and -0.1 (blue). The inflow perturbation is optimal at $T=180, \omega=0$ and $\left\|\boldsymbol{u}_{B}^{\prime}\right\|_{b}=$ $2 \times 10^{-2}$ and an external forcing is added to activate secondary instabilities.

the nonlinear optimal inflow disturbance would have been unsteady. The results show that the inflow distortion comprises streamwise velocity and vorticity. It interacts with the boundary layer starting from the leading edge of the plate, and the response are streaks which are prone to secondary instability. In the presence of additional free-stream forcing, the streaky base flow undergoes secondary instability and breaks down to turbulence.

\section{Conclusion}

The response of flow over a flat plate with a slender leading edge to free-stream perturbations was studied. The focus was placed on identifying the free-stream perturbations that are most effective at generating an energetic response inside the downstream boundary layer. Using an adjoint, both the linear and nonlinear scenarios were examined, and the inlet perturbations were restricted to being monochromatic in time. In the linear limit, the disturbance field remains monochromatic in time downstream, and all the spanwise wavenumbers remain decoupled. As the energy of the inflow disturbance is increased, the problem becomes nonlinear. The monochromatic restriction on the inflow was retained, so we can isolate the effect of every frequency at perturbing the boundary layer. However, higher harmonics develop downstream due to the nonlinear advection term in the Navier-Stokes equations. The spanwise spectral content of the disturbance in the nonlinear case is broadband, even at the inlet.

In the linear limit, the most effective inflow perturbations are steady and symmetric relative to the plate. Low-frequency inflow perturbations with $\omega<0.14$ can also induce a response within the boundary layer, while disturbances with $\omega>0.14$ are filtered by the mean shear. Appropriately scaled, the optimal inflow perturbation has a normalized spanwise wavenumber $\beta^{\prime} \equiv \beta / \sqrt{R e} \simeq 0.05$ that is insensitive to the Reynolds number over the range of parameters examined. The leading edge strengthens the streamwise vorticity, which subsequently leads to the amplification of streaks via the lift-up mechanism.

When the streak magnitude becomes appreciable, the flow becomes prone to secondary instabilities with streamwise wavenumbers $\sim O(1)$. The most unstable mode is symmetric relative to the high-speed streak and resides near the wall. 
By introducing body forcing in the simulation domain, within the unstable region, the secondary instability of the streaky flow can be activated and bypass transition is observed downstream.

In the present study, the inflow was restricted to be monochromatic in temporalfrequency space. This assumption enabled the study of the most effective components of the free-stream spectrum to interact with the boundary layer, nonlinearly, and to generate an energetic response. Future work should relax this assumption, and allow the inflow perturbation to include multiple frequency components. In that context, the objective function can be to promote transition to turbulence. The inflow disturbance would then include the essential elements to induce an energetic, and also unstable, boundary-layer distortion that undergoes laminar-to-turbulent transition at the upstream most streamwise location.

\section{Acknowledgements}

X.M. acknowledges financial support from the Engineering and Physical Sciences Research Council (EPSRC, grant number EP/M025039/1). T.A.Z. acknowledges support from the Office of Naval Research (ONR, grant number N00014-16-12542). B.W. acknowledges support from the Natural Science Foundation of China (NSFC, grant numbers 11502137, 11732010). Calculations were performed on HPC-Midlands funded by the Engineering and Physical Sciences Research Council (grant number EP/K000063/1).

\section{REFERENCES}

Andersson, P., Berggren, M. \& Henningson, D. S. 1999 Optimal disturbances and bypass transition in boundary layers. Phys. Fluids 11 (1), 134-150.

Andersson, P., Brandt, L., Bottaro, A. \& Henningson, D. S. 2001 On the breakdown of boundary layer streaks. J. Fluid Mech. 428, 29-60.

Asai, M., Minagawa, M. \& NishiokA, M. 2002 The instability and breakdown of a near-wall low-speed streak. J. Fluid Mech. 455, 289-314.

Balamurugan, G. \& MAndal, A. C. 2017 Experiments on localized secondary instability in bypass boundary layer transition. J. Fluid Mech. 817, 217-263.

Barkley, D., Blackburn, H. M. \& Sherwin, S. J. 2008 Direct optimal growth analysis for timesteppers. Intl J. Numer. Meth. Fluids 57, 1435-1458.

BiAncofiore, L., BRAndT, L. \& ZAKI, T. A. 2017 Streak instability in viscoelastic Couette flow. Phys. Rev. Fluids 2 (043304), 1-20.

Boiko, A. V., Westin, K. J. A., Klingmann, B. G. B., Kozlov, V. V. \& Alfredsson, P. H. 1994 Experiments in a boundary layer subjected to free stream turbulence. Part 2. The role of TS-waves in the transition process. J. Fluid Mech. 281, 219-245.

Bose, R. \& Durbin, P. A. 2016 Helical modes in boundary layer transition. Phys. Rev. Fluids 1, 073602.

BRANDT, L. \& DE LANGE, H. C. 2008 Streak interactions and breakdown in boundary layer flows. Phys. Fluids 20 (2), 024107.

Brandt, L., Schlatter, P. \& Henningson, D. S. 2004 Transition in boundary layers subject to free-stream turbulence. J. Fluid Mech. 517, 167-198.

Brandt, L., SipP, D., Pralits, J. O. \& MARQuet, O. 2011 Effects of base-flow variation in noise amplifiers: the flat-plate boundary layer. J. Fluid Mech. 687, 503-528.

Butler, K. M. \& FARRELL, B. F. 1992 Three-dimensional optimal perturbations in viscous shear flow. Phys. Fluids A 4 (8), 1637-1650.

Cossu, C. \& BRAndT, L. 2002 Stabilization of Tollmien-Schlichting waves by finite amplitude optimal streaks in the Blasius boundary layer. Phys. Fluids 14, L57. 
Fransson, J. H. M., Brandt, L., Talamelli, A. \& Cossu, C. 2005 Experimental study of the stabilization of Tollmien-Schlichting waves by finite amplitude streaks. Phys. Fluids 17, 054110 .

HACK, M. J. P. \& ZAKI, T. A. 2014 Streak instabilities in boundary layers beneath free-stream turbulence. J. Fluid Mech. 741, 280-315.

HACK, M. J. P. \& ZAKI, T. A. 2016 Data-enabled prediction of streak breakdown in pressure-gradient boundary layers. J. Fluid Mech. 801, 43-64.

Hultgren, L. S. \& Gustavsson, L. H. 1981 Algebraic growth of disturbances in a boundary layer. Phys. Fluids 24, 1000-1004.

Hunt, J. C. R. \& Durbin, P. A. 1999 Perturbed vortical layers and shear sheltering. Fluid Dyn. Res. 24, 375-404.

Jacobs, R. G. \& Durbin, P. A. 2001 Simulations of bypass transition. J. Fluid Mech. 428, 185-212.

Karniadakis, G. \& Sherwin, S. 2005 Spectral/HP Element Methods for Computational Fluid Dynamics. Oxford University Press.

Kendall, J. M. 1991 Studies on laminar boundary layer receptivity to free stream turbulence near a leading edge. In Boundary Layer Stability and Transition to Turbulence (ed. X. Reda), vol. 114, pp. 23-30. ASME-FED.

Klebanoff, P. S. 1971 Effect of freestream turbulence on the laminar boundary layer. Bull. Am. Phys. Soc. 16, 1323.

Landahl, M. T. 1975 Wave breakdown and turbulence. SIAM J. Appl. Maths 28 (4), 735-756.

Leib, S. J., Wundrow, D. W. \& Goldstein, M. E. 1999 Effect of free-stream turbulence and other vortical disturbances on a laminar boundary layer. J. Fluid Mech. 380, 169-203.

LiU, Y., ZAKI, T. A. \& DURBIN, P. A. 2008 a Boundary-layer transition by interaction of discrete and continuous modes. J. Fluid Mech. 604, 193-233.

LiU, Y., ZAKI, T. A. \& DURBIN, P. A. $2008 b$ Floquet analysis of secondary instability of boundary layers distorted by Klebanoff streaks and Tollmien-Schlichting waves. Phys. Fluids 20, 124102.

LUCHINI, P. 2000 Reynolds-number-independent instability of the boundary layer over a flat surface: optimal perturbations. J. Fluid Mech. 404, 289-309.

Mandal, A. C., Venkatakrishnan, L. \& DeY, J. 2010 A study on boundary-layer transition induced by free-stream turbulence. J. Fluid Mech. 660, 114-146.

Mans, J., De Lange, H. C. \& van Steenhoven, A. A. 2007 Sinuous breakdown in a flat plate boundary layer exposed to free-stream turbulence. Phys. Fluids 19, 088101.

Mao, X., Blackburn, H. M. \& Sherwin, S. J. 2013 Calculation of global optimal initial and boundary perturbations for the linearised incompressible Navier-Stokes equations. J. Comput. Phys. 235, 258-273.

Mao, X., Blackburn, H. M. \& Sherwin, S. J. 2015 Nonlinear optimal suppression of vortex shedding from a circular cylinder. J. Fluid Mech. 775, 241-265.

MaO, X., Zaki, T. A., Blackburn, H. M. \& Sherwin, S. J. 2017 Transition induced by linear and nonlinear perturbation growth in flow past a blade. J. Fluid Mech. 820, 604-632.

Matsubara, M. \& Alfredsson, P. 2001 Disturbance growth in boundary layers subjected to free-stream turbulence. J. Fluid Mech. 430, 149-168.

Monokrousos, A., Åkervik, E., Brandt, L. \& Henningson, D. S. 2010 Global three-dimensional optimal disturbances in the blasius boundary-layer flow using time-steppers. J. Fluid Mech. 650, 181-214.

Nagarajan, S., Lele, S. K. \& Ferziger, J. H. 2007 Leading-edge effects in bypass transition. J. Fluid Mech. 572, 471-504.

NolAN, K. P. \& WALSH, E. J. 2012 Particle image velocimetry measurements of a transitional boundary layer under free stream turbulence. J. Fluid Mech. 702, 215-238.

NOLAN, K. P. \& ZAKI, T. A. 2013 Conditional sampling of transitional boundary layers in pressure gradients. J. Fluid Mech. 728, 306-339.

Phillips, O. M. 1969 Shear-flow turbulence. Annu. Rev. Fluid Mech. 1, 245-264.

Schmid, P. J. \& Henningson, D. S. 2000 Stability and Transition in Shear Flows. Springer. 
Schrader, L.-U., Brandt, L., Mavriplis, C. \& Henningson, D. S. 2010 Receptivity to freestream vorticity of flow past a flat plate with elliptic leading edge. J. Fluid Mech. 653, $245-271$.

TAYLOR, G. I. 1939 Some recent developments in the study of turbulence. In Proceedings Fifth International Congress for Applied Mechanics (ed. J. P. Den Hartog \& H. Peters), pp. 294-310. Wiley.

VAUGhan, N. J. \& ZAKI, T. A. 2011 Stability of zero-pressure-gradient boundary layer distorted by unsteady Klebanoff streaks. J. Fluid Mech. 681, 116-153.

Westin, K. J. A., Bakchinov, A. A., Kozlov, V. V. \& Alfredsson, P. H. 1998 Experiments on localized disturbances in a flat plate boundary layer. Part 1 . The receptivity and evolution of a localized free stream disturbance. Eur. J. Mech. (B/Fluids) 17, 823-846.

Westin, K. J. A., Boiko, A. V., Klingmann, B. G. B., Kozlov, V. V. \& Alfredsson, P. H. 1994 Experiments in a boundary layer subjected to free stream turbulence. Part 1. Boundary layer structure and receptivity. J. Fluid Mech. 281, 193-218.

ZAKI, T. A. 2013 From streaks to spots and on to turbulence: exploring the dynamics of boundary layer transition. Flow Turbul. Combust. 91, 451-473.

ZAKi, T. A. \& Durbin, P. A. 2005 Mode interaction and the bypass route to transition. J. Fluid Mech. 531, 85-111.

ZAKI, T. A. \& DURBIN, P. A. 2006 Continuous mode transition and the effects of pressure gradients. J. Fluid Mech. 563, 357-358.

ZAKI, T. A. \& SAHA, S. 2009 On shear sheltering and the structure of vortical modes in singleand two-fluid boundary layers. J. Fluid Mech. 626, 111-147.

Zuccher, S., Bottaro, A. \& Luchini, P. 2006 Algebraic growth in a blasius boundary layer: nonlinear optimal disturbances. Eur. J. Mech. (B/Fluids) 25, 1-17. 